\title{
Functional Th1-oriented T follicular helper cells that infiltrate human breast cancer promote effective adaptive immunity
}

\author{
Grégory Noël, ${ }^{1}$ Mireille Langouo Fontsa, ${ }^{1}$ Soizic Garaud, ${ }^{1}$ Pushpamali De Silva, ${ }^{1}$ Alexandre de Wind, ${ }^{2}$ Cert G. Van den Eynden, ${ }^{1,3}$ \\ Roberto Salgado, ${ }^{3}$ Anaïs Boisson, ${ }^{1}$ Hanne Locy, ${ }^{4}$ Noémie Thomas, ${ }^{1}$ Cinzia Solinas, ${ }^{1}$ Edoardo Migliori, ${ }^{1}$ Céline Naveaux, \\ Hugues Duvillier, ${ }^{1,5}$ Sophie Lucas, ${ }^{6}$ Ligia Craciun, ${ }^{2}$ Kris Thielemans, ${ }^{4}$ Denis Larsimont, ${ }^{2}$ and Karen Willard-Gallo ${ }^{1}$ \\ 1'Molecular Immunology Unit, and '2Department of Pathology, Institut Jules Bordet, Université Libre de Bruxelles, Brussels, Belgium. ${ }^{3}$ Department of Pathology, CZA Ziekenhuizen, Sint-Augustinus Campus, \\ Wilrijk, Belgium. ${ }^{4}$ Laboratory of Molecular and Cellular Therapy, Vrije Universiteit Brussel, Brussels, Belgium. ${ }^{5}$ Flow Cytometry Facility, Institut Jules Bordet, Université Libre de Bruxelles, Brussels, Belgium. \\ ${ }^{6}$ de Duve Institute, Université Catholique de Louvain, Brussels, Belgium.
}

\begin{abstract}
We previously demonstrated that tumor-infiltrating lymphocytes (TIL) in human breast cancer sometimes form organized tertiary lymphoid structures (TLS) characterized by CXCL13-producing T follicular helper (Tfh) cells. The present study found that CD4+ Tfh TIL, CD8 ${ }^{+}$TIL, and TIL-B, colocalizing in TLS, all express the CXCL13 receptor CXCR5. An ex vivo functional assay determined that only activated, functional Th1-oriented Tfh TIL (PD-1 ${ }^{\text {hi } I C O S^{\text {int }}}$ phenotype) provide help for immunoglobulin and IFN- $\gamma$ production. A functional Tfh TIL presence signals an active TLS, characterized by humoral (immunoglobulins, Ki-67+ TIL-B in active germinal centers) and cytotoxic (GZMB+CD8+ and GZMB+CD68 ${ }^{+}$TIL plus Th1 gene expression) immune responses. Analysis of active versus inactive TLS in untreated patients revealed that the former are associated with positive clinical outcomes. TLS also contain functional T follicular regulatory (Tfr) TIL, which are characterized by a CD25+CXCR5 ${ }^{+}$CARP ${ }^{+}$FOXP3 ${ }^{+}$ phenotype and a demethylated FOXP3 gene. Functional Tfr inhibited functional Tfh activities via a glycoprotein A repetitions predominant (GARP)-associated TCF- $\beta$-dependent mechanism. The activity of tumor-associated TLS was dictated by the relative balance between functional Tfh TIL and functional Tfr TIL. These data provide mechanistic insight into TLS processes orchestrated by functional Th1-oriented Tfh TIL, including TIL-B and CD8+ TIL activation and immunological memory generation. Tfh TIL, regulated by functional Tfr TIL, are an expected key target of PD-1/PD-L1 blockade.
\end{abstract}

\section{Introduction}

Adaptive and innate antitumor immune responses and their exploitation in immunotherapy are recognized as key defenses in the fight against cancer. Across a diversity of tumor types, data support the important link between higher densities of tumor-infiltrating lymphocytes (TIL) and better clinical outcomes (1). In breast cancer (BC), TIL density is predictive and prognostic for the $\mathrm{HER}_{2}{ }^{+}$and triple-negative (TN) subtypes (2), both characterized by higher frequencies of extensively infiltrated tumors compared with the luminal subtypes (3).

Globally, TIL contain a mixture of adaptive and innate immune cells typically dominated by $\mathrm{T}$ cells, but with varied subpopulation balances between tumor types and individual patients. Further, their migratory nature triggers dynamic changes in TIL positioning and balance in regions within the tumor microenvironment (TME). Isolated TIL are more frequently observed in the tumor bed, while TIL accumulations are commonly located in the stroma as aggregates or tertiary lymphoid structures (TLS) (4). TLS have been detected in

Authorship note: GN and MLF contributed equally to this work. Conflict of interest: The authors have declared that no conflict of interest exists. Copyright: @ 2021, American Society for Clinical Investigation. Submitted: May 8, 2020; Accepted: August 11, 2021; Published: October 1, 2021 Reference information: J Clin Invest. 2021;131(19):e139905. https://doi.org/10.1172/JCl139905. a wide range of solid tumors, with evidence supporting their functionality as mini-lymph nodes at these chronic inflammatory sites $(5,6)$. An increasing number of studies, including our work on BC, report that tumor-associated TLS with active germinal centers (GC) are linked with positive clinical outcomes $(4,7-9)$ and responses to immunotherapy (10-12). Previous work also revealed that TLS exist in different maturation states $(7,8,13)$.

Our BC studies first associated TLS with the specific presence of $\mathrm{T}$ follicular helper ( $\mathrm{Tfh}$ ) cells in tumors with higher infiltrates (4, 14). Tfh cells are specialized $\mathrm{CD} 4^{+} \mathrm{T}$ cells that were first recognized for their role in helping memory and antibody-producing B cell differentiation in the GC (15). In addition to providing T-dependent B cell help, Tfh cells can also guide migration and promote GC formation under normal and pathological conditions. We identified a Tfhlike $\mathrm{CD}^{+} \mathrm{CXCR}^{-} \mathrm{BC}$ TIL subpopulation (TfhX13) as the principal source of CXCL13, the chemoattractant that drives CXCR5+ lymphocyte migration and contributes to TLS formation $(4,14)$. These specialized TfhX13 cells are similarly present in normal human tonsils where, in contrast with those in BC, they are consistently CXCR5 ${ }^{+}$ (14). CXCL13-producing CD4 ${ }^{+}$CXCR5 $\mathrm{T}$ cells in blood (called peripheral helper $\mathrm{T}$ [Tph] cells) have also been identified in patients with rheumatoid arthritis (RA) $(16,17)$. Interestingly, we also detected CXCL13-producing CD8 ${ }^{+}$TIL at lower frequencies, but comingling with $\mathrm{CD}^{+}$TIL and TIL-B in BC tissues (14). Subsequently, 
CXCL13-producing CD8 ${ }^{+} \mathrm{PD}-1^{+} \mathrm{T}$ cells (18) and TGF- $\beta$-dependent CXCL13-producing $\mathrm{CD} 103^{+} \mathrm{CD}^{+} \mathrm{T}$ cell TIL (19) were identified in lung cancer and melanoma, respectively, although CXCR5 expression was not examined. Finally, CXCL13 gene-expression studies have demonstrated its prognostic and predictive value for a variety of chronic inflammatory diseases, including viral infections (20), autoimmune diseases (21), and cancer, where it is consistently a strong component of gene signatures $(4,22)$.

The influence of CXCR5 ${ }^{-}$TfhX13 TIL on TIL recruitment and TLS induction/formation in BC (14) led us to investigate CXCR5 ${ }^{+}$ TIL subpopulations migrating in response to CXCL13. Our previous study of TIL-B determined that, while all TIL-B differentiation stages infiltrate $\mathrm{BC}$, tumors with high TIL are characterized by GC and antibody-secreting TIL-B in correlation with Tfh cells, all localized in TLS (23). In this study, we identify $\mathrm{CD} 4^{+} \mathrm{CXCR}^{+} \mathrm{Tfh} \mathrm{TIL}$, $\mathrm{CD}^{+}{ }^{+} \mathrm{CXCR}^{+} \mathrm{TIL}$, and CXCR5 ${ }^{+}$TIL-B as characteristic of TLS-positive BC. We further show that only Tfh TIL in vivo educated in active TLS have the capacity to provide T-dependent B cell help and produce IFN- $\gamma$ in an ex vivo functional assay. Tumors maintaining a balance of active (versus inactive) TLS are distinguished by functional Th1-oriented Tfh TIL, a higher proportion of effector versus regulatory TIL, and a better prognosis.

\section{Results}

CXCR5 $5^{+}$TIL are primarily localized in human BC-associated TLS. $\mathrm{CXCR}^{+} \mathrm{TIL}$, the targets of CXCL13 recruitment, were analyzed in a cohort of invasive ductal BC patients $(n=168$; clinicopathological parameters in Supplemental Table 1; supplemental material available online with this article; https://doi.org/10.1172/ JCI139905DS1) scored for TIL and TLS (as in ref. 3). In line with previous studies, infiltrating lymphocytes increased in BC tissues, with higher TIL densities (TIL/mg of tissue) more frequently observed in HER2 ${ }^{+}$and TN BC (Supplemental Figure 1A) $(3,24)$. Examination of CXCR5 ${ }^{+}$TIL subpopulations revealed significantly lower frequencies of $\mathrm{CD} 4^{+} \mathrm{CXCR} 5^{+} \mathrm{TIL}$ and higher frequencies of $\mathrm{CD}^{+} \mathrm{CXCR}^{+}$TIL and $\mathrm{CD} 19^{+} \mathrm{CXCR}^{+}{ }^{+} \mathrm{TIL}-\mathrm{B}$ compared with what was found in human tonsils (mean $[\mu]$ values in Figure 1A; gating strategies in Supplemental Figure 1B and Supplemental Table 2).

$\mathrm{CD} 4^{+}$TIL contain a mixture of functionally and phenotypically diverse subpopulations, with $\mathrm{CD} 4^{+} \mathrm{CXCR} 5^{+} \mathrm{T}$ cells classically designated $\mathrm{Tfh}$, now known to include both Tfh and $\mathrm{T}$ follicular regulatory (Tfr) cells. A summary of the $\mathrm{CD}^{+} \mathrm{T}$ cell subpopulation phenotypes used in this study is provided in Table 1. Infiltration by Tfh TIL, CD8 ${ }^{+}$CXCR5 ${ }^{+}$TIL, and CXCR5 $5^{+}$TIL-B was highly correlated (Figure 1B) and independent of the BC subtype (Supplemental Figure 1C). The global CXCR5 $5^{+}$TIL densities in fresh tissues (determined by flow cytometry) were well correlated with TLS scored on dual CD3/CD20 chromogenic IHC-stained (cIHCstained) tissues from the same patient (Figure 1C and Supplemental Table 3 ). In contrast, TIL aggregates containing T cell TIL $\left(\mathrm{CD}^{+}\right.$and $\left.\mathrm{CD} 8^{+}\right)$and TIL-B $\left(\mathrm{CD} 2 \mathrm{O}^{+}\right)(23)$ were not correlated with $\mathrm{CXCR}^{+}$TIL. These data suggest an important association exists between the coinfiltration of CXCR5 ${ }^{+}$TIL subpopulations and the formation and/or presence of a TLS.

TLS were next identified and analyzed on sequential tissue sections from TIL-positive BC $(n=7)$ using CD3/CD20 cIHC (Figure 1D) and CXCR5, CD4 or CD8, and CD20 immunofluorescent
(IF) labeling (Figure 1E and Supplemental Figure 1, D and E). The majority of $\mathrm{CD}^{+}$TIL, CD8 ${ }^{+}$TIL, and TIL-B within a TLS were $\mathrm{CXCR}^{+}$(purple T cells, yellow B cells), while those outside (blue T cells, green B cells) were predominantly CXCR5-. This colocalization of CXCR5 ${ }^{+}$TIL within a TLS is shown for additional tumors (Supplemental Figure 1F) and validated by DAPI staining and pixel analysis (CD4/CD20 shown in Supplemental Figure 1G). Based on our previous and current data, the CXCR5 ${ }^{+}$TIL detected by flow cytometry are reasoned to be predominantly resident in $\mathrm{BC}$-associated TLS. Colocalization of $\mathrm{CD} 4{ }^{+} \mathrm{CXCR}^{+} \mathrm{T}$ cells and $\mathrm{CD} 2 \mathrm{O}^{+} \mathrm{CX}-$ $\mathrm{CR}^{+} \mathrm{B}$ cells was also seen in tonsils (Supplemental Figure $1 \mathrm{H}$ ). In contrast with those in $\mathrm{BC}$, tonsillar $\mathrm{CD} 8^{+} \mathrm{CXCR} 5^{+} \mathrm{T}$ cells were at extremely low frequencies, although when present, they tended to be near other $\mathrm{CXCR}^{+}$cells. These data confirm that $\mathrm{CXCR} 5^{+} \mathrm{TIL}$ (Tfh TIL, CD8 ${ }^{+}$TIL, and TIL-B) are recruited to BC-associated TLS where they can interact with one another and additional TME cells in their efforts to generate antitumor immune responses.

Functional Tfh TIL infiltrate human BC with active immune responses. Our previous $\mathrm{BC}$ studies of $\mathrm{CD} 4^{+} \mathrm{TIL}$ identified CXCL13-producing TfhX13 TIL by their distinctive CXCR5-PD$1^{\text {hi ICOS }}{ }^{\text {int }}$ phenotype (Table 1; note: tonsillar TfhX13 are CXCR5 $\left.{ }^{+}\right)(4$, 14). The present study expanded our analyses by quantifying PD-1 and ICOS on distinct CXCR5 and CXCR5 ${ }^{+} \mathrm{CD} 4^{+}$TIL subpopulations $\left(\mathrm{CD} 4^{+} \mathrm{CD} 25^{+} \mathrm{T}\right.$ cells, which are predominantly Tregs, were excluded) (Figure 2A). These data confirm that TfhX13 TIL are PD- ${ }^{\text {hi ICO- }}$ $\mathrm{S}^{\text {int }}$ and primarily CXCR5 ${ }^{-}(14)$, with a smaller population of $\mathrm{CXCR}^{+}$ Tfh TIL, also PD- $1^{\text {hi ICOS }}{ }^{\text {int }}$, detected in some tumors. Both of these subpopulations were most frequently found in HER $2^{+}$or TN BC and linked with higher global TIL (Supplemental Figure 2A). The majority of CXCR5 ${ }^{+}$Tfh TIL were PD- $1^{\text {lo/int }}$ ICOS $^{\text {lo }}$, with their CXCR5 ${ }^{-}$counterparts primarily PD- ${ }^{10}{ }^{\text {ICOS }}{ }^{\text {lo }}$ Th TIL.

Comparative analysis of tonsils with TIL identified key differences in the 3 distinct CXCR5 subpopulations detected within the $\mathrm{CD} 4^{+} \mathrm{CD} 25^{-} \mathrm{T}$ cell compartment (Supplemental Figure 2B). Tonsils contained (a) CXCR5 ${ }^{-}$non-Tfh cells (principally Th, hereafter referred to as such) with a PD- $1^{\text {lo }} \mathrm{ICOS}^{\mathrm{lo}}$ phenotype, (b) CXCR5 ${ }^{+} \mathrm{Tfh}$ cells that express PD- 1 and ICOS across a range from low to intermediate, and (c) CXCR5 $5^{\text {hi }}$ Tfh cells with a PD- $1^{\text {hi ICOS }}{ }^{\text {int }}$ phenotype

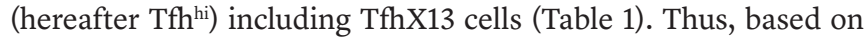
PD-1 and ICOS expression levels, Tfh TIL were similar to CXCR5 ${ }^{+}$ tonsillar Tfh cells, while TfhX13 TIL corresponded to tonsillar Tfh ${ }^{\text {hi }}$ cells (14). Tfh TIL do not express substantial amounts of Bcl6, in contrast with tonsillar Tfh cells, although both were principally of a memory T cell phenotype (Supplemental Figure 2, B and C). The latter suggests that Tfh TIL have previously been primed and differentiated, but does not affirm their tumor antigen specificity.

Th, Tfh, and TfhX13 TIL plus the 3 tonsillar subpopulations detailed above were sorted from $\mathrm{CD} 4^{+} \mathrm{CD} 25^{-}$cells for geneexpression analysis. Similarly to tonsillar Tfh cells, TfhX13 and Tfh TIL abundantly expressed CXCL13 and IL21 gene transcripts, with the highest levels detected in TN BC (Figure 2B and Supplemental Figure 2, B and C). Interestingly, unlike tonsillar Tfh cells, TfhX13 TIL additionally expressed higher levels of the canonical IFNG (IFN- $\gamma$ ) gene, suggesting a Th1 orientation. TfhX13 TIL also expressed higher levels of the IL1O and MAF genes compared with Th TIL. IF labeling (consecutive sections of Figure 1E and Supplemental Figure $1 \mathrm{~F}$ ) confirmed the localization of $\mathrm{CD} 4^{+} \mathrm{CXCR} 5^{+} \mathrm{PD}-1^{+}$ 
A

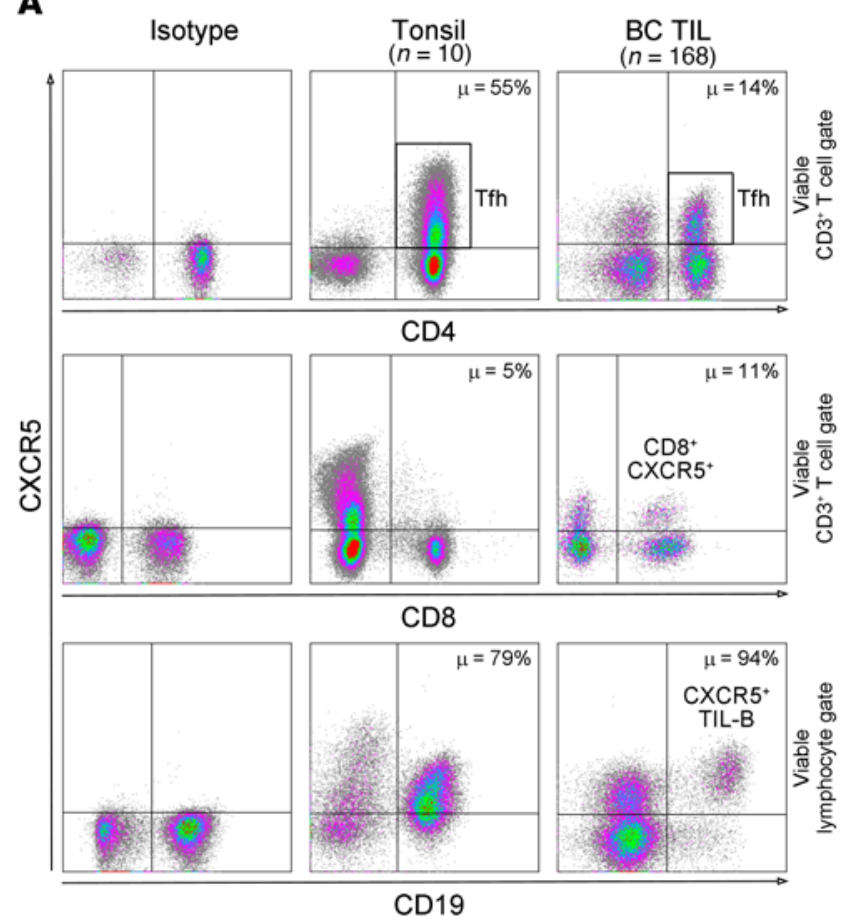

C

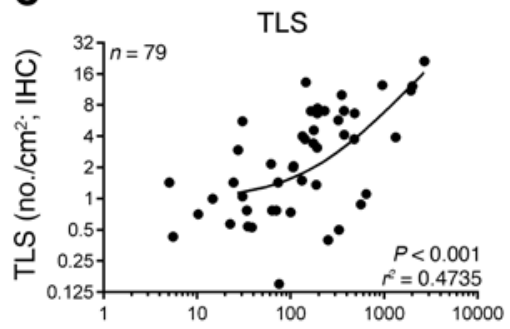

Aggregates

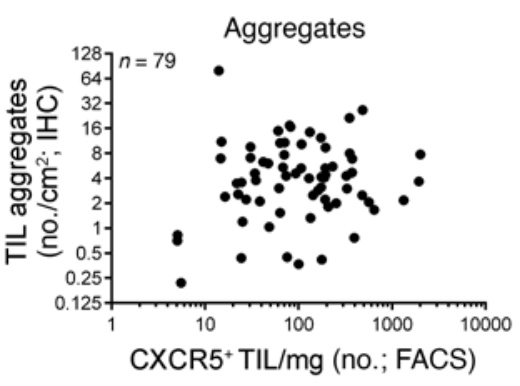

D

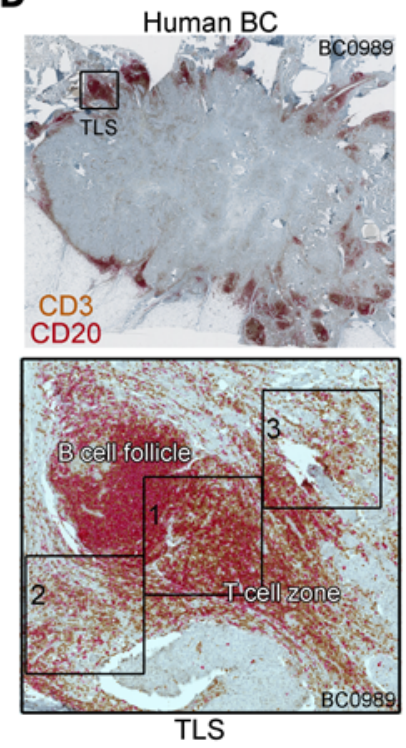

B
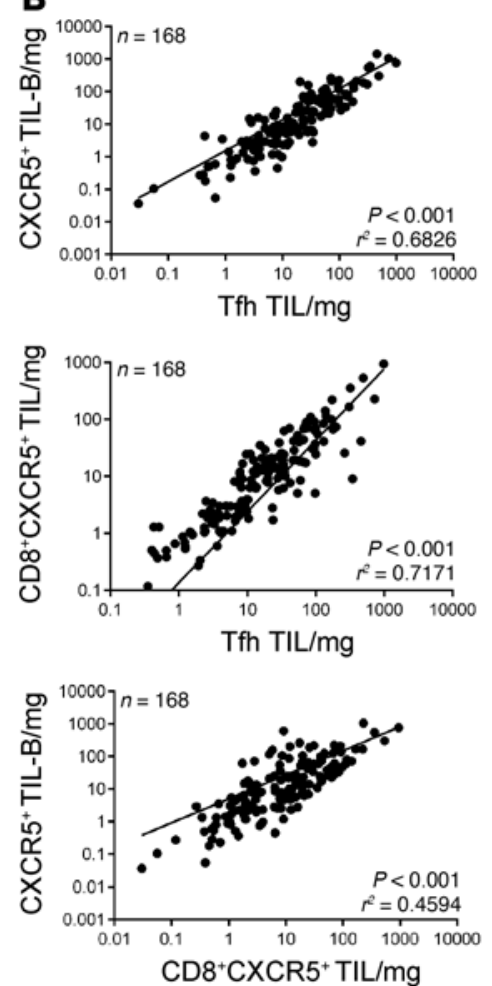

E
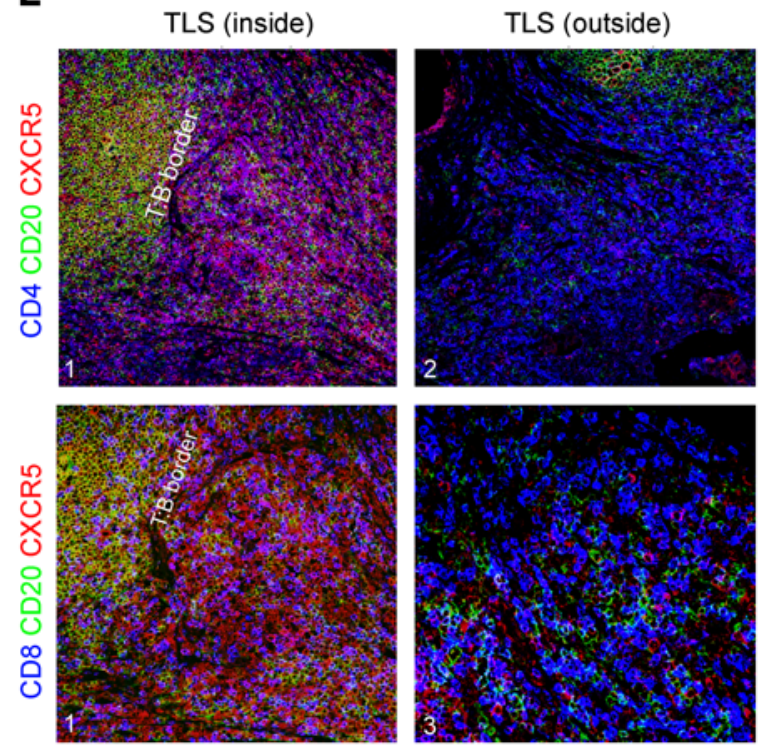

Figure 1. CXCR5 ${ }^{+}$TIL are primarily localized in human BC-associated TLS. (A) Lymphocytes from fresh tissue homogenates (ref. 29) of human tonsils ( $n=$ 10) or BC ( $n=168$; luminal A [LumA; $n=82$ ], luminal B [LumB; $n=36$ ], HER2 ${ }^{+}[n=24]$, TN $[n=26]$ ) were immunophenotyped (flow cytometry). The mean frequency $(\mu)$ of $C X C R 5^{+}$cells within the $C D 4, C D 8$, and $C D 20$ subpopulations is shown. Upper plots: $C D 3^{+} C D 4^{+} C D 45^{+} T$ cells; middle plots: $C D 3^{+} C D 8^{+} C D 455^{+}$ T cells; lower plots: CD19+CD45+ B cells. (B) TIL densities in human BC tumors ( $n=168$; number of TIL/mg of tumor; flow cytometry) for CXCR5 ${ }^{+}$Tfh TIL, CXCR5 ${ }^{+}$TIL-B, and CD8 ${ }^{+}$CXCR5 ${ }^{+}$TIL correlated with one another (linear regression analysis). (C) TIL densities (CXCR5 ${ }^{+}$Tfh TIL + CD8 ${ }^{+}$CXCR5 $5^{+}$TIL + CXCR5 $5^{+}$ TIL-B/mg of tumor; flow cytometry) were correlated with TIL aggregates or TLS scored on dual CD3/CD2O (brown/red) cIHC-stained FFPE tissue sections (as described in refs. 3, 39) from the same tumor ( $n=79$; linear regression analysis). (D) Upper panel: representative dual CD3/CD20 clHC (TN BC 0989); lower panel: zoom images of 3 TLS areas: 1, inside a TLS showing the T cell zone and B cell follicle; 2 and 3 , focused on areas outside the TLS. Upper panel: magnification $\times 3.5$; lower panel magnification: $\times 35$. (E) IF staining of a consecutive tissue section showing the 3 areas in $\mathbf{D}$. Left panels: colocalization of CD20+CXCR5 ${ }^{+}$TIL-B (yellow) and CD4+CXCR5 ${ }^{+}$Tfh TIL (purple) or CD8 ${ }^{+}$CXCR5 ${ }^{+}$TIL (purple) inside the TLS. Magnification $\times 60$. Right panels: CXCR5- CD4 ${ }^{+} /$ $\mathrm{CD8}^{+}$(blue) or $\mathrm{CD}_{2} \mathrm{O}^{+}$(green) TIL outside the TLS. The T:B border is the junction between the T cell zone and the B cell follicle. Upper right magnification $\times 100$; lower right magnification $\times 150$. 


\section{Table 1. Phenotypes of CD4+ helper T cell subpopulations in human breast tumors and tonsils}

\begin{tabular}{|c|c|c|}
\hline \multicolumn{3}{|c|}{ CD4 ${ }^{+}$subpopulations: CXCR5 expression ${ }^{A}$} \\
\hline $\begin{array}{l}\text { Tfh TIL (Tfh and Tfr) } \\
\text { Other CD4 }{ }^{+} \text {TL (TfhX13, Th and Tregs) } \\
\text { Tfh Tonsils (Tfh, Tfr and TfhX13) } \\
\text { Other CD4+ Tonsils (Th and Tregs) }\end{array}$ & $\begin{array}{l}\text { CXCR5 }^{+} \\
\text {CXCR5 } \\
\text { CXCR5 } \\
\text { CXCR5 }\end{array}$ & \\
\hline \multicolumn{3}{|l|}{ Tfh: $\mathrm{CD}^{+} \mathrm{CD}^{-} 5^{-}$} \\
\hline $\begin{array}{l}\text { Functional }{ }^{\mathrm{B}} \text { Tfh TIL (Th1-oriented) } \\
\text { Nonfunctional }{ }^{\mathrm{B}} \text { Tfh TIL } \\
\text { TfhX13 TIL } \\
\text { Tfh Tonsils } \\
\text { Tfh } \\
\text { Tfi Tonsils (includes TfhX13) }\end{array}$ & 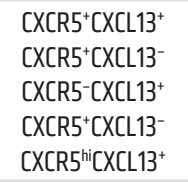 & 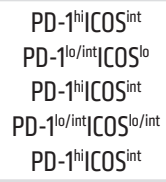 \\
\hline \multicolumn{3}{|l|}{ Tfr: $\mathrm{CD}^{+}{ }^{\mathrm{CD}} 25^{+} \mathrm{CXCR}^{+}$} \\
\hline $\begin{array}{l}\text { Functional }{ }^{E} \text { Tfr TIL } \\
\text { Nonfunctional Tfr TIL } \\
\text { Functional Tfrint Tonsils } \\
\text { Nonfunctional Tfri tonsils }\end{array}$ & $\begin{array}{l}\text { Foxp3 }^{+} \text {CARP }^{+} \\
\text {Foxp }^{+} \text {GARP } \\
\text { Foxp3 }{ }^{+} \text {GARP } \\
\text { Foxp3-CARP- }\end{array}$ & 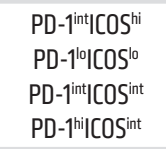 \\
\hline \multicolumn{3}{|c|}{ Other $\mathrm{CD}^{+}$subpopulations: $\mathrm{CD4}^{+} \mathrm{CXCR5}^{-}$} \\
\hline $\begin{array}{l}\text { Th TIL } \\
\text { Activated Treg TIL }\end{array}$ & $\begin{array}{c}{\mathrm{CD} 25^{-}}^{-} \\
\mathrm{CD} 25^{+} \mathrm{Foxp}^{3} \mathrm{CARP}^{+}\end{array}$ & $\begin{array}{l}\text { PD-1'10|COS'lo } \\
\text { PD-1 } 1^{\text {int }} \mid \mathrm{COS}^{\text {hi }}\end{array}$ \\
\hline
\end{tabular}

AExpression levels of markers shown are based on flow cytometric analysis. ${ }^{B}$ Functionality is based on data from the in vitro Tfh functional assay. ${ }^{\mathrm{C}}$ Abbreviated as TON in figures. ${ }^{\mathrm{D}}$ Expression can be heterogeneous in the same tonsil (Supplemental Figure 2A) or between tonsils. EFunctionality is based on data from the in vitro functional assay using Tfr from tonsils.

TIL principally in TLS, both in the T cell zone and the B cell follicle (Figure 2C and Supplemental Figure 2D). These data identify 2 important TIL subpopulations whose presence is consistently associated with TLS-positive human BC: (a) conventional CXCR5 ${ }^{+}$Tfh TIL and (b) CXCR5-CXCL13-producing TfhX13 TIL, both characteristically expressing PD-1, ICOS, IL21, and CXCL13.

Th, Tfh, and TfhX13 TIL were tested for their ability to help human splenic B cells produce immunoglobulins using the ex vivo functional assay methodology depicted in Figure 2D. Fresh tissues from 9 BC specimens were preselected for high TIL densities $(>250$ $\mathrm{TIL} / \mathrm{mg}$ tissue) and the presence of Tfh TIL, which are specifically associated with TLS $(4,14)$. Tfh (9/9), TfhX13 (3/9), and Th (9/9) TIL were sorted directly ex vivo on the basis of PD-1 and ICOS expression (Figure 2A), with these subpopulations subsequently activated ex vivo in the Tfh functional assay (Supplemental Figure 2E). Remarkably, only Tfh TIL with a PD- ${ }^{\text {hi }}$ ICOS $^{\text {int }}$ phenotype ex vivo (4/9) were proficient B cell helpers for IgG and IgA production ex vivo and thus are identified as functional Tfh TIL (Figure 2, E and F, and Table 1). Th (non-Tfh) TIL from 2 of 4 of the BC containing functional Tfh TIL also helped moderate amounts of IgA but not IgG production. TfhX13 TIL did not provide help despite the presence of functional Tfh TIL in 3 tumors in which TfhX13 TIL were present. Functional Tfh TIL were detected in the HER2 ${ }^{+}(2 / 3)$ and TN (2/3), but not in luminal A (0/3), BC. Tfh from tonsils, used as a control, were the most efficient helper $\mathrm{CD}^{+} \mathrm{T}$ cell subpopulation for IgG and IgA production (Supplemental Figure 2B; note: like TfhX13 TIL, tonsillar Tfh ${ }^{\text {hi }}$ are not functional).

Analysis of cytokines secreted in assay supernatants revealed that both Th and Tfh TIL, sorted from BC containing functional Tfh
TIL, produced higher amounts of IFN- $\gamma$, IL-2, and LT $\alpha$ proteins than tumors containing nonfunctional Tfh TIL (Figure 2G). This contrasts with tonsils, in which Th cells were the principal IFN- $\gamma$, IL-2, and LT $\alpha$ producers (Supplemental Figure 2B). These data are reinforced by the significant increases in gene transcripts for CXCL13, $I L 21$, and IFNG as well as the transcription factors BCL6 and MAF detected in functional Tfh TIL compared with their nonfunctional counterparts (Figure $2 \mathrm{G}$ ). Additionally, a significant $\left({ }^{*} P<0.05 ;{ }^{* *} P\right.$ $<0.01$ ) percentage of functional (but not nonfunctional) Tfh TIL were positive for CXCL13 protein, albeit at somewhat lower levels than TfhX13 TIL. Finally, IL-10 protein produced by Th and, to a lesser extent, Tfh (similar to tonsils) was again found only in BC with functional Tfh TIL, suggesting that Tregs specifically infiltrate or differentiate in the TME of these immunologically active tumors. Overall, these data suggest that when functional Tfh TIL are resident in an active TLS, their cellular interactions and secreted gene products help promote a Th1 microenvironment that generates antitumor immune responses.

The proportion of Tfr TIL in human BC is associated with TLS activities. Successful immune responses are produced when the balance favors effector and cytotoxic TIL over regulatory cells in quantity and activity within a local microenvironment. Tregs, known for maintaining normal tissue homeostasis and their ability to retract immune responses, frequently infiltrate solid tumors. Evidence is emerging that other specialized regulatory cells targeting specific immune subpopulations can also accumulate in tumors. Here, we investigated Tfr cells whose known function is to control Tfh help for GC-mediated humoral immunity (25).

Based on CD25 and CXCR5 positivity/negativity, we identified 4 distinct $\mathrm{CD}^{+}{ }^{+}$TIL subpopulations: (a) Tfr cells (18\% of $\mathrm{CD}^{+} \mathrm{CX}-$ CR5 ${ }^{+}$TIL), (b) Tregs (19\% of CD4 $4^{+}$CXCR5 $^{-}$TIL), (c) Tfh cells, and (d) the remaining Th cells (Figure 3A and Supplemental Table 4). Foxp3 protein expression and FOXP3 gene demethylation, the latter required for its stable expression (26), were predominantly detected in Tfr TIL and Tregs isolated from peripheral blood mononuclear cells (PBMCs) or TIL (Figure 3B; CD25 and Foxp3 expression were notably different in tonsillar $\mathrm{CD}^{+}{ }^{+} \mathrm{T}$ cells, Supplemental Figure $3 \mathrm{~A}$ ). Tfr and Treg TIL had similar expression patterns, significantly different from those of Th cells, that were characterized by high immunosuppressive (IL-35 in Tfr but not Treg and glycoprotein A repetitions predominant [GARP]), checkpoint (CD39, TIGIT and CTLA4), and transcription factor (IKZF2, IKZF4 and FOXP3) genes (Figure $3 \mathrm{C})$. Some of these genes were specific to the TME in comparison with tonsillar $\mathrm{CD} 4^{+} \mathrm{T}$ cell subpopulations (Supplemental Figure $3 \mathrm{~B}$ ). CD4 and Foxp3 cIHC staining showed close contact between CD4 ${ }^{+-}$ Foxp $^{+}$and $\mathrm{CD}^{+}{ }^{+}$oxp $^{-}$TIL, principally in the $\mathrm{T}$ cell zone and $\mathrm{T}: \mathrm{B}$ border of TLS (Figure 3D). Interestingly, some CD $4^{+} \mathrm{Foxp}^{+} \mathrm{TIL}$ also interact with $\mathrm{CD}^{+} \mathrm{Foxp}^{-}$TIL in the B cell follicle (zoom 1), while in the stroma, they were mostly scattered (zoom 2). Our data showing that the majority of $\mathrm{CD} 4^{+} \mathrm{T}$ cells in a TLS are $\mathrm{CXCR} 5^{+}$(Figure 1 and Supplemental Figure 1) support the view that the $\mathrm{CD} 4^{+} \mathrm{Foxp} 3^{+} \mathrm{TIL}$ here (Figure 3D) are Tfr cells and not Tregs.

Analysis of expression of GARP, a TGF- $\beta 1$-docking protein that is upregulated on activated Tregs (27), revealed a higher percentage of Tfr TIL were GARP ${ }^{+}$compared with Treg TIL, with similar findings for Tfr from tonsils (Supplemental Figure 3, C and D). GARP ${ }^{+}$ Tregs and Tfr TIL both expressed higher levels of CD25 and ICOS 
A
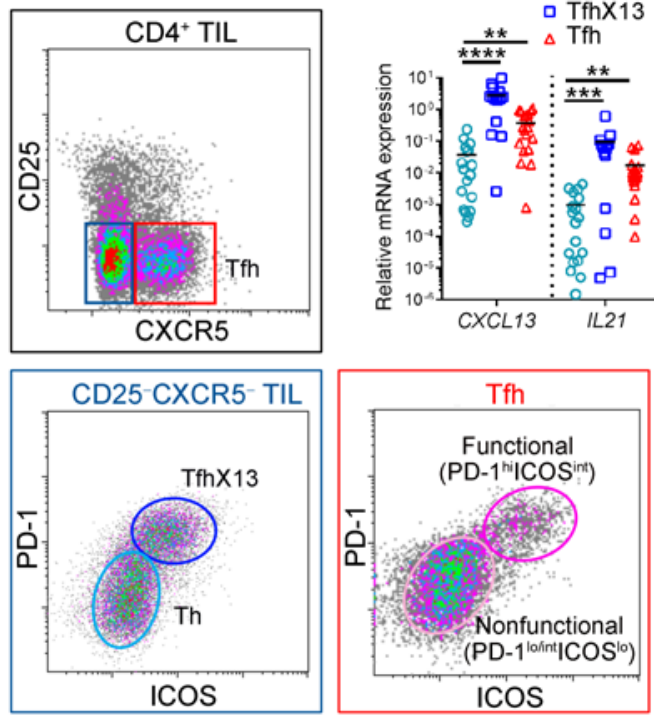

$\mathbf{E}$
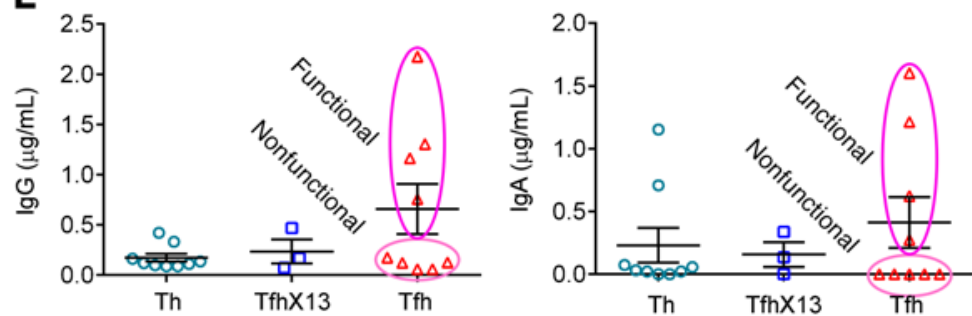

C

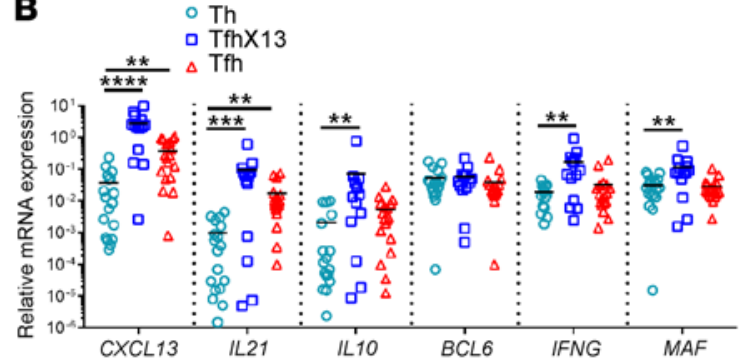

D Tfh functional assay

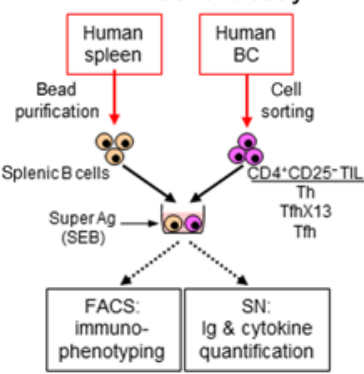

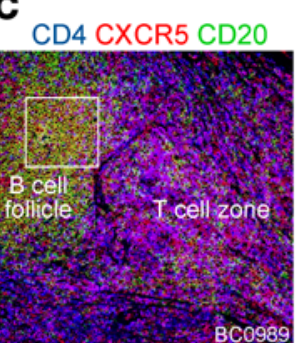
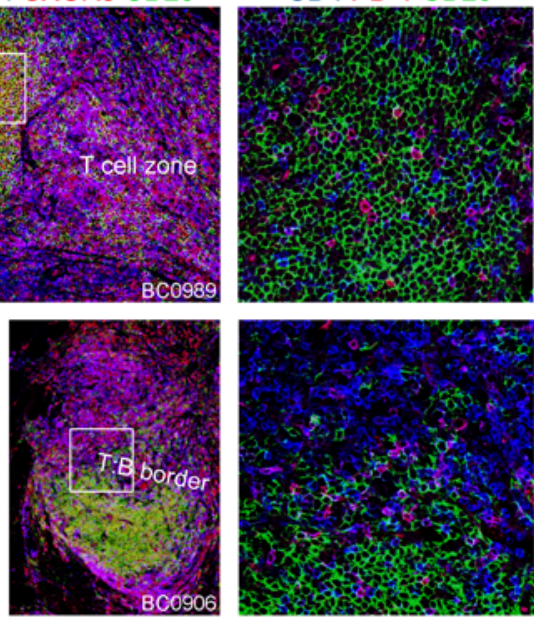

F $\quad$ BC Tfh TIL functionality

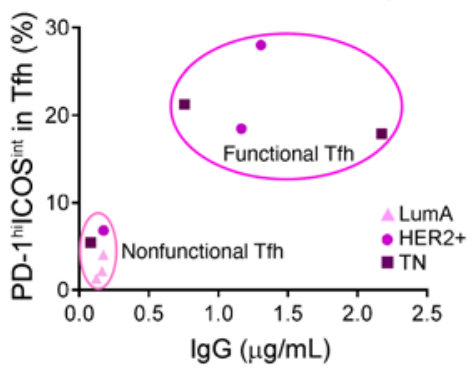

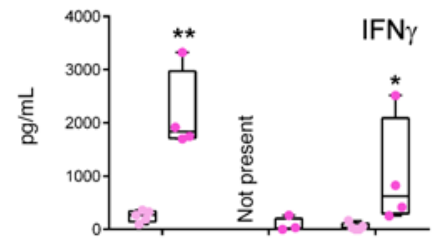
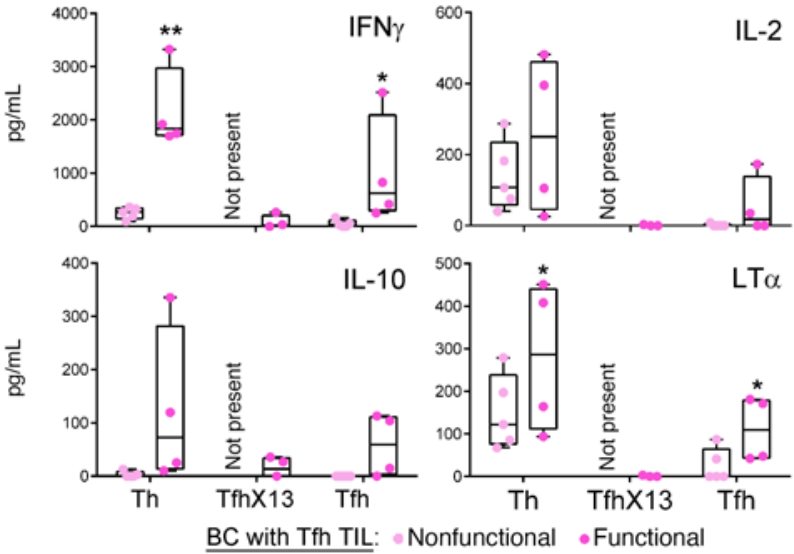
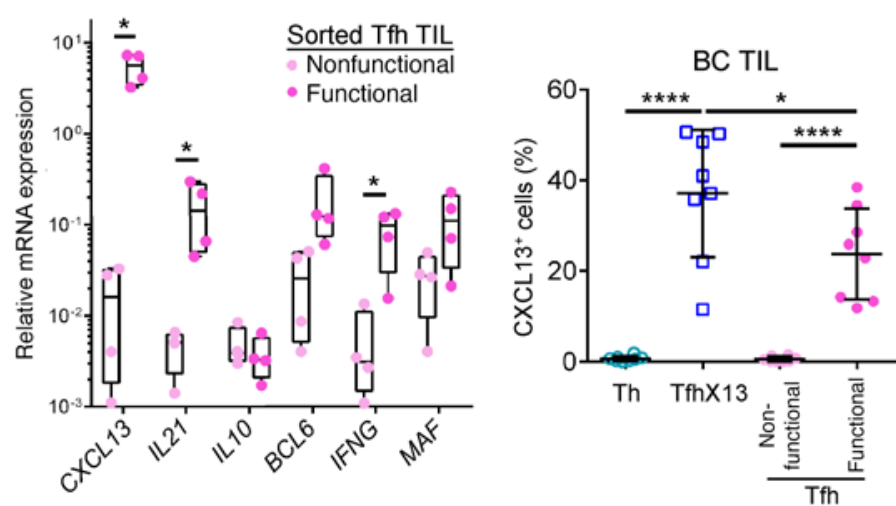

Figure 2. Functional Tfh TIL infiltrate tumors with active immune responses. (A) CD4+ TIL analyzed by flow cytometry for CD25, CXCR5, ICOS, and PD-1

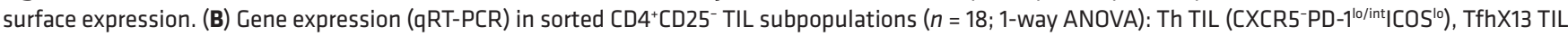

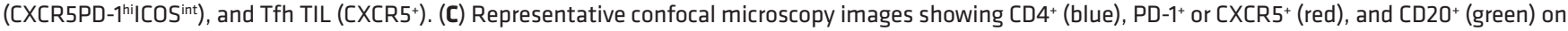
consecutive sections of the tumors in Figure $1 \mathrm{E}$ (BC 0989) and S1F (BC 0906). A zoomed image inside the TLS B cell follicle (BC0989) or at the T:B border (BC 0906) is shown. Upper left panel magnification: $\times 60$; upper right $\times 170$; lower left $\times 45$; lower right $\times 170$. (D) Flow chart for the Tfh functional assay: 3 subpopulations of CD4+CD25- TIL (Th, Tfh and TfhX13 TIL) are sorted from BC homogenates and activated ex vivo with SEB in cocultures with human splenic B cells. Cells are harvested at day 3 for immunophenotyping and culture supernatants at day 7 for immunoglobulin and cytokine quantification. (E) Assay supernatants ( $n=9$ for Th TIL and Tfh TIL; $n=3$ for TfhX13 TIL) analyzed for IgG (left panel) and IgA (right panel) produced in assay cocultures with Th, TfhX13, or Tfh

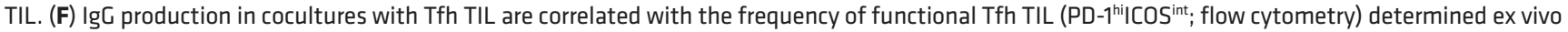
(i.e., at the time of sorting for the Tfh functional assay). Left panel: cytokines/chemokines in the assay supernatants containing Th, TfhX13, or Tfh TIL; the 3 subpopulations are subdivided into nonfunctional $(n=5)$ and functional Tfh TIL ( $n=4$; Student's $t$ test). Middle panel: gene-expression analysis (qRT-PCR) of sorted nonfunctional (PD-1/1/intICOS ${ }^{10}$ ) and functional (PD- $1^{\text {hil ICOS }}$ int) Tfh TIL ( $n=4$; Student's $t$ test). Right panel: intracytoplasmic CXCL13 staining (\% positive cells) in the Th, TfhX13, or Tfh TIL subpopulations (flow cytometry; $n=8$; 1-way ANOVA). ${ }^{*} P<0.05 ;{ }^{* *} P<0.01 ;{ }^{* * *} P<0.001$; ${ }^{* * *} P<0.0001$. 
A

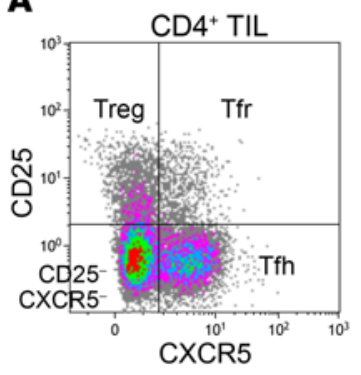

B

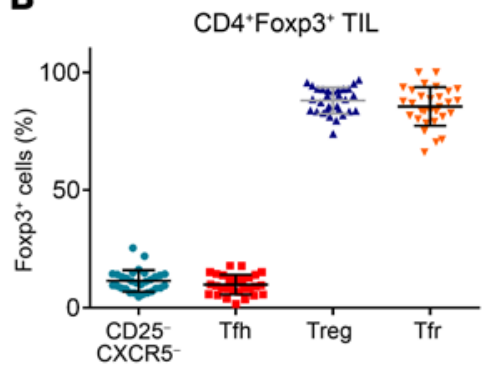

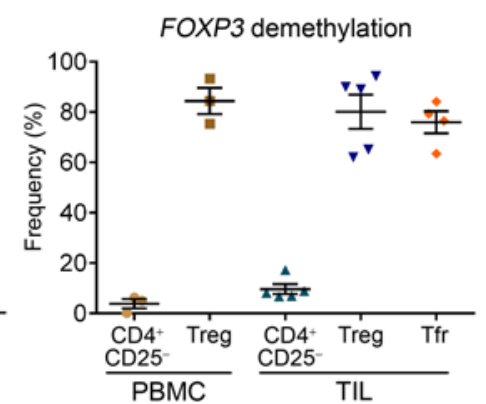

C

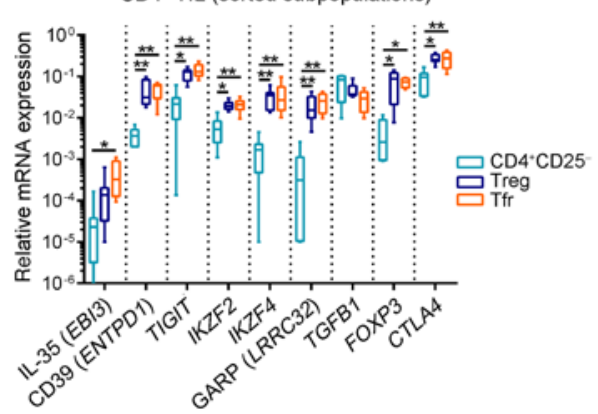

D

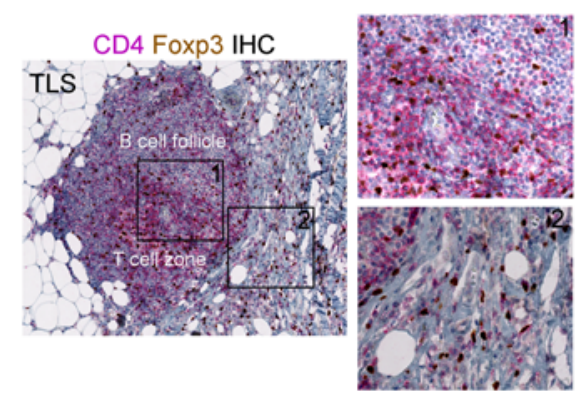

E Tfr functionality (Tonsils:

Functional Tfrint $-T_{f r}{ }^{\text {hi) }}$
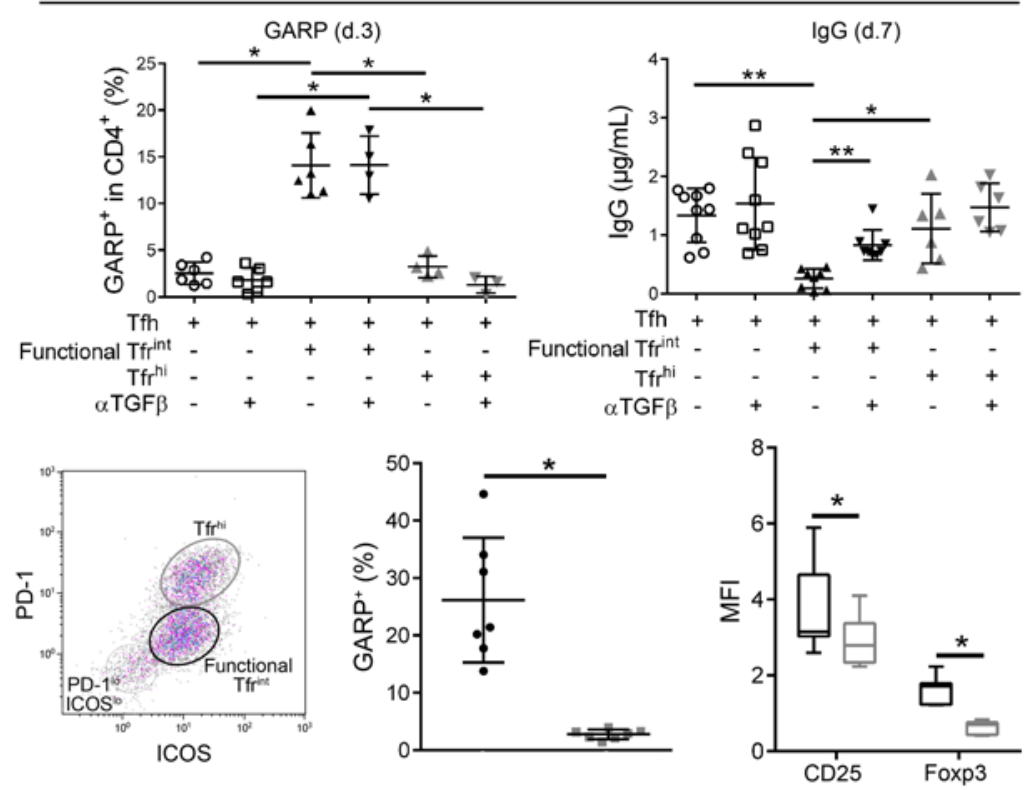

$\mathbf{F}$
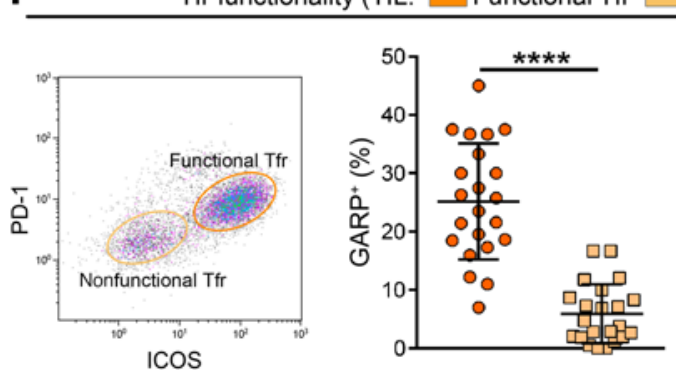

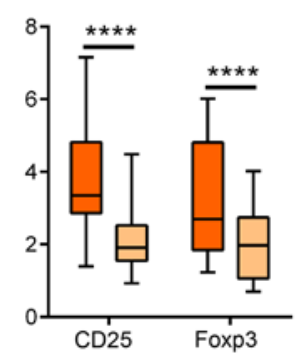

Figure 3. The proportion of Tfr TIL in human BC is associated with TLS activity. (A) Four distinct CD4 ${ }^{+}$TIL subpopulations are identified based on CD25 and CXCR5 surface expression (flow cytometry): (a) Tfr (CD25 CX$\mathrm{CR}^{+}$), (b) Tregs (CD25+CXCR5-), (c) Tfh (CD25-CXCR5 ${ }^{+}$), and (d) the remaining Th (CD25CXCR5 ${ }^{-}$). (B) Left: nuclear Foxp3 expression in CD4 ${ }^{+}$TIL was positive in $11 \%$ of CD25-CXCR5-, 9\% of Tfh, $88 \%$ of Tregs, and $86 \%$ of Tfr (flow cytometry; $n=30)$. Right: FOXP3 gene demethylation in sorted TIL: CD4+CD25- $(10 \%+)$, Treg $(80 \%+)$ and $\operatorname{Tfr}(76 \%+)(n=5)$. PBMC sorted from healthy donors: CD4+CD25 $(4 \%+)$, Tregs $(84 \%+)(n=3)$. (C) Gene-expression analysis (qRT-PCR) of the sorted CD4+ TIL subpopulations in A (1-way ANOVA). (D) Left: representative dual CD4/Foxp3 (red/brown) clHC-staining of human BC. Upper right: zoomed image inside a TLS; lower right: zoomed image just outside a TLS; both showing Tfh TIL (red membrane, blue nuclei) and Tfr/Treg TIL (red membrane, brown nuclei; location dependent for Tfr or Treg). The TLS shown is $600 \mu \mathrm{m}$ wide. Magnification: $\times 40$ (left panel); $\times 100$ (right panels). (E) Sorted functional $\operatorname{Tfr}\left(\mathrm{PD}-1^{\text {int }}\right)$ and $\operatorname{Tfr} P D-1^{\text {hi }}$ from tonsils activated in Tfh functional assay cocultures with tonsillar Tfh cells plus or minus an anti-TCF- $\beta$ antibody ( $n=9$; 1 -way ANOVA). Upper left: $\mathrm{CARP}^{+}$cell frequencies among $\mathrm{CD} 4^{+}$cells (on assay day 3); upper right: IgG production (on assay day 7). Lower left: tonsillar Tfr subpopulations sorted on ICOS and PD-1 (flow cytometry); lower middle: GARP ${ }^{+}$ cell frequencies and lower right: CD25 and Foxp3 MFI for functional Tfrint and Tfrhi tonsillar cells ( $n=7$; Student's $t$ test). (F) Left: Tfr TIL subpopulations sorted on ICOS and PD-1 (flow cytometry); middle: GARP+ cell frequencies; right: CD25 and Foxp3 MFI for functional Tfrit and Tfr ${ }^{\text {hi }}$ TIL subpopulations ( $n=21$; Student's $t$ test). ${ }^{*} P<$ $0.05 ;{ }^{* *} P<0.01 ;{ }^{* * *} P<0.0001$. 
than their GARP $^{-}$counterparts, signaling their activation in tumors. This contrasts with tonsils, in which higher CD25 and ICOS were detected on only a subset of potentially reactive cells in this tissue. Because insufficient numbers of Tfr were available from fresh BC tissues, their effect on Tfh functionality was tested using 2 Tfr phenotypes: Tfr ${ }^{\text {int }}\left(\mathrm{PD}-1^{\text {int }} \mathrm{ICOS}^{\text {int }}\right)$ and $\mathrm{Tfr}^{\mathrm{hi}}\left(\mathrm{PD}-1^{\text {hi }} \mathrm{ICOS}^{\text {int }}\right)$ sorted from tonsils with enough $\mathrm{CD}^{+} \mathrm{Foxp}^{+} \mathrm{GARP}^{+} \mathrm{T}$ cells (Figure $3 \mathrm{E}$ ). Only tonsillar Tfrint, which are selectively Foxp $3^{+}$and $\mathrm{GARP}^{+}$, prohibited Tfh from helping B cells produce IgG and therefore are termed functional Tfrint. Low concentrations of TGF- $\beta 1$ blocked Tfh-dependent $\mathrm{B}$ cell differentiation, while an anti-TGF- $\beta$ antibody reduced functional $\mathrm{Tfr}^{\text {int }}$ suppressive activity, confirming their regulatory nature and suggesting TGF- $\beta$ dependence (similar to Tregs; Supplemental Figure 3E). To our knowledge, this is the first classification of functional tonsillar Tfr cells showing their $\mathrm{CD} 4^{+} \mathrm{CD} 25^{+} \mathrm{CXCR} 5^{+} \mathrm{Fox}-$ $\mathrm{p3}^{+} \mathrm{GARP}^{+} \mathrm{PD}-\mathrm{1}^{\text {int }} \mathrm{ICOS}^{\text {int }}$ phenotype (Supplemental Table 5).

A comparison of functional $\mathrm{Tfh}^{\text {int }}$ from tonsils with PD- $1^{\text {int }} \mathrm{I}-$ $\mathrm{COS}^{\text {hi }}$ Tfr TIL revealed that the latter also expressed higher levels of GARP, CD25, and Foxp3 compared with PD- $1^{\text {lo }}$ ICOS ${ }^{\text {lo }}$ Tfr TIL (Figure 3F). Based on their $\mathrm{CD}^{+} \mathrm{CD} 25^{+} \mathrm{CXCR} 5^{+} \mathrm{Foxp}^{+} \mathrm{GARP}^{+}$ phenotype, we characterized the PD- $1^{\text {int }} \mathrm{ICOS}^{\text {hi }}$ subpopulation as functional Tfr TIL (Figure 3F and Supplemental Figure 3C), with functional Tfr TIL and tonsillar Tfr ${ }^{\text {int }}$ both the more active subpopulations in their respective tissues. Supporting this notion, functional Tfr TIL were more frequently detected in HER2 ${ }^{+}$and TN compared with luminal BC (Supplemental Figure 3F). Further, when functional Tfr TIL were present, they were correlated with functional Tfh TIL in all BC subtypes except luminal A (Supplemental Figure 3G). The Tfh TIL to Tfr TIL ratios in BC, which we reason to be associated with TLS, are similar to those recently shown for Tfh and Tfr in the T cell zones of human mesenteric lymph nodes (28).

The balance offunctional Tfh to functional Tfr TIL governs humoral immune responses in human $B C$. The above data suggest that, as Tfh and Tfr TIL commingle in a TLS, the relative balance of effector to regulatory TIL dynamically determines the specific immune activities functioning in the TME. To test this hypothesis, the presence of functional Tfh TIL relative to characteristics of high TIL BC was evaluated. Immunoglobulin concentrations in supernatants from primary BC ( $n=71$; ref. 29$)$ were significantly higher in $\mathrm{HER}^{+}$(IgG and $\operatorname{IgM})$ and $\mathrm{TN}(\operatorname{IgG})$, the most frequent TIL-high BC subtypes (3), compared with normal breast tissue (Supplemental Figure 4A). Functional Tfh TIL densities were significantly correlated with IgG concentrations in $\mathrm{TN}$, with a similar trend observed in HER2 ${ }^{+} \mathrm{BC}$ (Figure 4A; no correlation found for IgA or IgM, data not shown).

The location of functional Tfh TIL within the TLS was examined in $\mathrm{HER}^{+}$and $\mathrm{TN} \mathrm{BC}$ (including the $6 \mathrm{HER} 2^{+} / \mathrm{TN}$ tumors shown in Figure 2). TLS-associated B cell follicles were identified using dual CD3/CD20 cIHC with a consecutive tissue section dual PD-1/Ki-67 cIHC stained (Figure 4B). In the B cell follicle, PD-1+ cells were scored as a surrogate for functional Tfh TIL and Ki- $67^{+}$ cells as a surrogate for GC TIL-B (together with their morphology; ref. 23). IF multiplex IHC (mIHC) confirmed the positioning of $\mathrm{CD}^{+}{ }^{+} \mathrm{PD}-1^{+}$functional Tfh TIL with CD $20^{+} \mathrm{Ki}-67^{+}$TIL-B in GCpositive TLS together with their relative absence in those lacking a GC (Supplemental Figure 4B). A significant correlation was observed between the presence or absence of functional Tfh TIL $\left(\mathrm{PD}-1^{+}\right)$and GC TIL-B $\left(\mathrm{Ki}-67^{+}\right)$in $76 \mathrm{~B}$ cell follicles (TLS $\pm \mathrm{GC}$;
HER2 ${ }^{+} /$TN BC, $\left.n=19\right)$ scored using cIHC (Figure 4C). Quantification of PD- $1^{+} \mathrm{CD} 4^{+}$and $\mathrm{CD} 2 \mathrm{O}^{+} \mathrm{Ki}-67^{+}$TIL on mIHC-stained BC $(n=$ 11) validated the direct correlation between functional Tfh TIL and GC TIL-B (Supplemental Figure 4C). Further, these data confirmed the robust link between functional Tfh TIL and GC TIL-B previously detected by flow cytometry $(14,23)$ while establishing their colocalization site as GC-positive TLS-associated B cell follicles. This suggests that important interactions between TLS-resident functional Tfh TIL and GC TIL-B are required for Ig production.

The impact of Tfr TIL on active humoral immune responses was evaluated by correlating the functional $\mathrm{Tfh} /$ functional $\mathrm{Tfr}$ TIL ratio with IgG and IgA production across BC subtypes $(n=71)$. The functional Tfh/Tfr ratios were significantly correlated with humoral immunity using Ig concentrations as a barometer (Figure 4D) and were clearly superior to functional Tfh TIL alone (Figure 4A). Interestingly, these ratios were similar to those recently shown for Tfh and Tfr cells in the T cell zone of human mesenteric lymph nodes (28). In BC, our data suggest that as functional Tfh and Tfr TIL commingle in the TLS, their relative balance controls the magnitude of active humoral immune responses.

A CD4/CD20/PD-1/ICOS/Ki-67/Foxp3 mIHC panel was used to examine Tfh and Tfr TIL contacts in the TME (Figure 4E and Supplemental Figure 4D). ICOS was included as an additional marker because its expression is significantly higher on functional Tfh and Tfr TIL compared with their nonfunctional counterparts (Supplemental Figure 4E). Further, comparing functional Tfh with functional Tfr revealed higher ICOS expression on the latter, suggesting that these subpopulations may compete for ICOS-L. Quantitative analysis of ICOS ${ }^{+}$functional Tfr TIL, principally located in the T cell zone, that touch either Tfh, functional Tfh, TIL-B, or other Tfr TIL revealed that functional Tfr most frequently contact functional $\mathrm{Tfh}$ in TLS with a GC. In non-GC TLS, the opposite was true, with more functional Tfr touching PD-1- Tfh TIL in the T cell zone (Figure $4 \mathrm{E}$ and Supplemental Figure 4F). Finally, the total Tfh/total Tfr TIL and functional Tfh/functional Tfr TIL ratios revealed a significantly higher ratio only for the latter in TLS with a GC compared with those without (Figure 4F). These data suggest that TIL entering the $\mathrm{T}$ cell zone are carefully monitored and selected for access to the $\mathrm{B}$ cell follicle where they are further controlled for their maturation and differentiation stage, again similar to observations reported in human mesenteric lymph nodes (28). These close interactions highlight the importance Tfr TIL play in controlling Tfh TIL-dependent TIL-B differentiation in the TLS.

The balance between functional Tfh and Tfr TIL mediates CD ${ }^{+}$ immune responses in human $B C$. The production of Th1 cytokines by functional Tfh TIL in the assay supernatant suggests that their presence in active TLS can drive resident $\mathrm{CXCR} 5^{+}$effector $\mathrm{T}$ cells, including CD8 ${ }^{+} \mathrm{TIL}$, to generate Th1-oriented responses. Closer examination of CD8 ${ }^{+}$TIL identified 3 subpopulations based on PD-1 and ICOS expression levels: PD- ${ }^{\text {lo }} \mathrm{ICOS}^{\mathrm{lo}}, \mathrm{PD}-\mathrm{1}^{\mathrm{int}} \mathrm{ICOS}^{\mathrm{lo}}$, and PD- $\mathbf{1}^{\text {hi }} \mathrm{ICO}-$ $\mathrm{S}^{\text {int }}$ (Figure 5A). The majority were CD8 ${ }^{+} \mathrm{CXCR} 5$ TIL with TLS-resident $\mathrm{CD}^{+} \mathrm{CXCR}^{+}$TIL averaging $13 \%$ in $\mathrm{PD}-1^{\text {lo }}, 17 \%$ in $\mathrm{PD}-1^{\text {int }}$, and $6 \%$ in PD- ${ }^{\text {hi }}$ subpopulations (Supplemental Table 6). Genes associated with $\mathrm{CD}^{+} \mathrm{T}$ cell functions $(18,30,31)$ were comparatively analyzed in subpopulations sorted on PD-1 and ICOS (Figure 5B; note: TIL were too limited to sort individual subpopulations of CXCR5 ${ }^{+}$ and $\mathrm{CXCR}^{-}$). $\mathrm{CD} 8^{+}$PD-1 ${ }^{\text {lo }}$ and PD-1 ${ }^{\text {int }}$ TIL expressed higher levels 

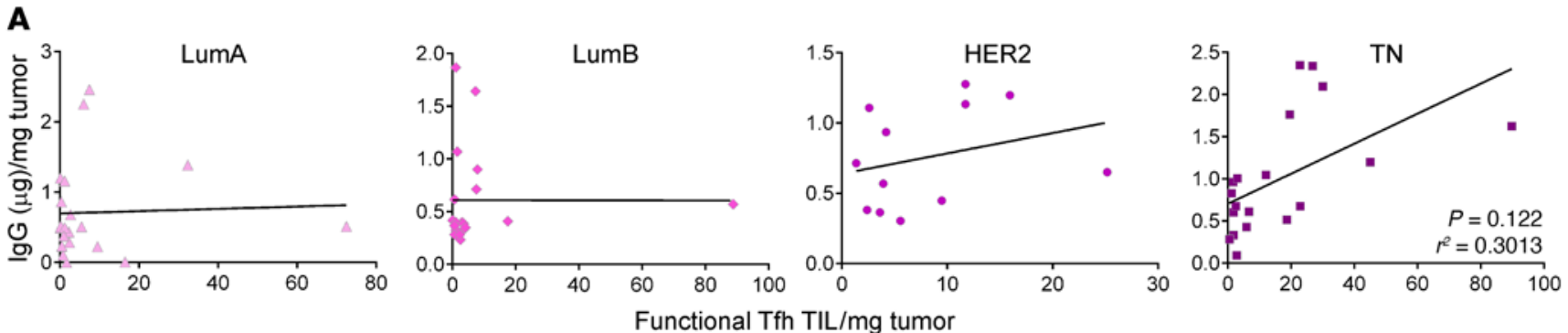

B

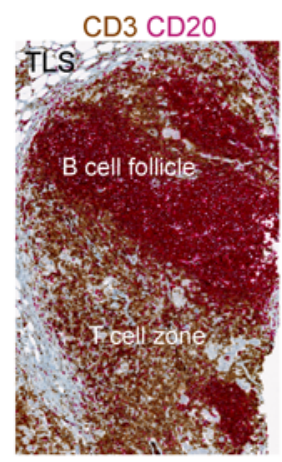

D
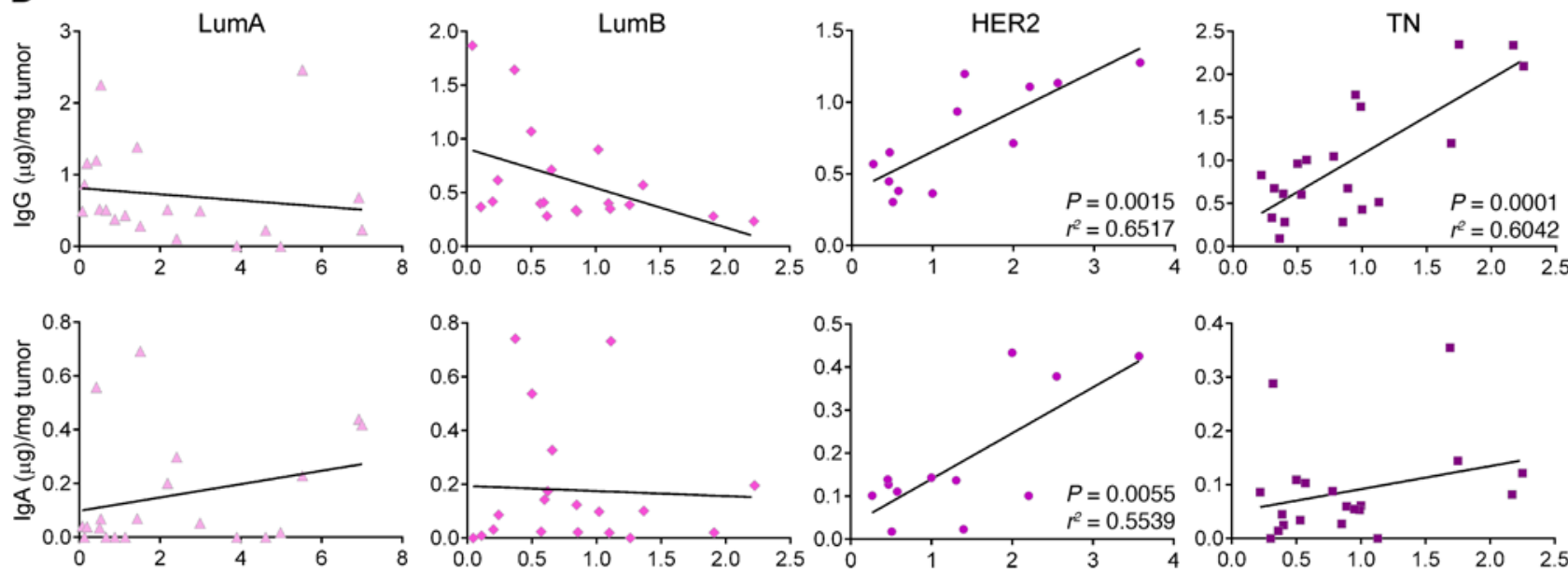

Functional Tfh TIL/Functional Tfr TIL (ratio)

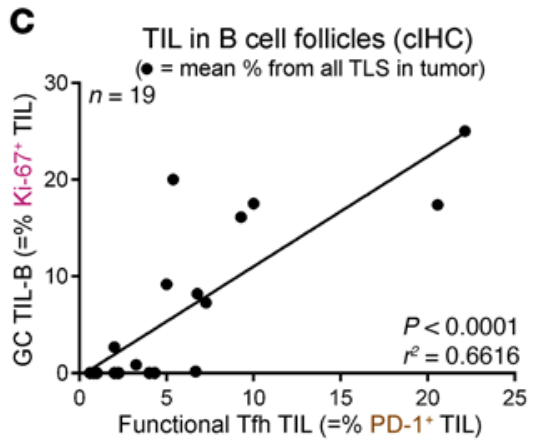

E
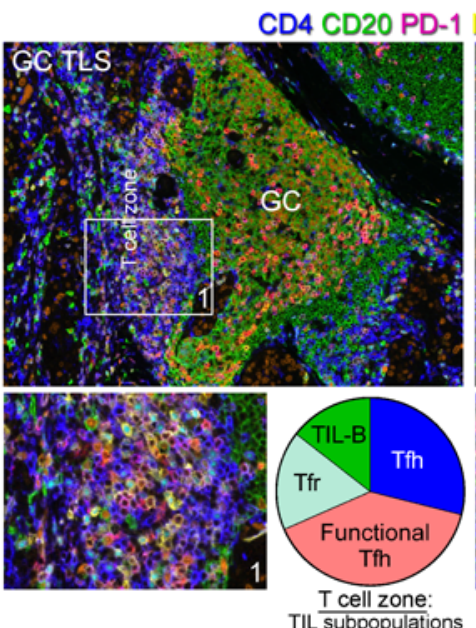

TIL subpopulations

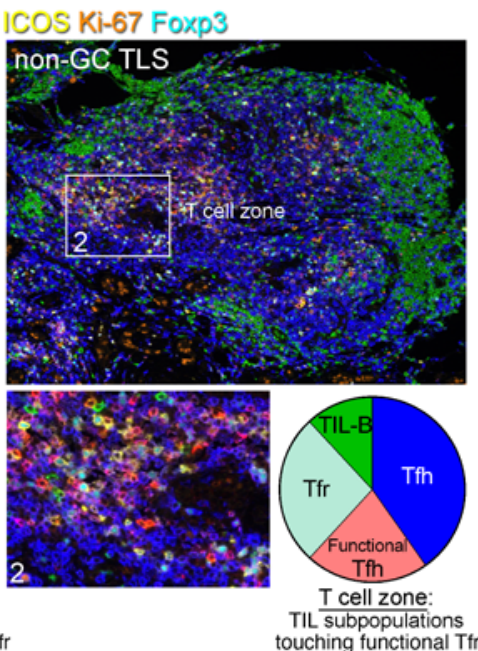

$\mathbf{F}$
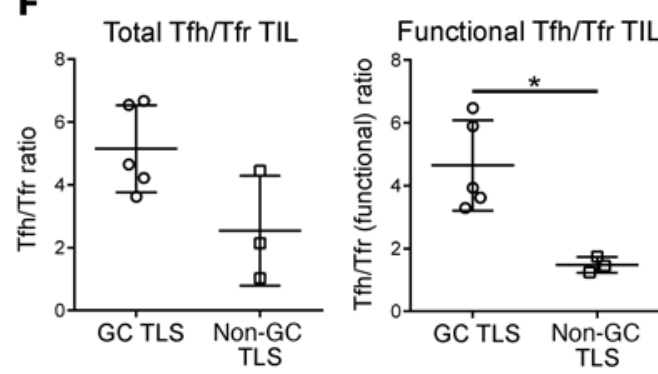
Figure 4. The balance of functional Tfh to functional Tfr TIL governs humoral immune responses in human BC. (A) Correlation between the density of functional Tfh TIL (no./mg tumor; flow cytometry) and IgG ( $\mu \mathrm{g} /$ mg tumor in the fresh tissue supernatant; $n=71$; LumA [ $n=20]$, LumB $[n=$ 19], HER2 $2^{+}[n=12]$, and TN $[n=20]$ ). (B) Representative FFPE sections (TNBC 1068 shown) were $\mathrm{CIHC}$ stained for scoring. Left: dual CD3/CD20 (brown/ red) for T cell zones and B cell follicles; middle: dual PD-1/Ki-67 (brown/red); consecutive sections; right: zoomed image of the B cell follicle. The TLS is $1000 \mu \mathrm{m}$ wide. Magnification: $\times 35$ (left panels); $\times 140$ (right panel). (C) Correlation between the frequency of $\mathrm{PD}-1^{+}$cells (functional Tfh TIL surrogate) and $\mathrm{Ki}-67^{+}$cells (GC TIL-B surrogate) in TLS-associated B cell follicles stained and scored as in B. Each point shows the mean score for all TLS in a given tumor. (D) The ratio of functional Tfh to functional Tfr TIL (flow cytometry) correlated with supernatant Igs (A). Upper panels: IgG $(\mu \mathrm{g}) / \mathrm{mg}$ tumor; lower panels: IgA ( $\mu \mathrm{g}) / \mathrm{mg}$ tumor ( $n=71$; LumA $[n=20]$, LumB $[n=19]$, HER2 ${ }^{+}[n=$ 12], TN $[n=20]$ ). (E) mIHC for CD2O (green), Ki-67 (orange), CD4 (blue), PD-1 (red), ICOS (yellow), and Foxp3 (cyan) on a representative FFPE section (2 TLS; TNBC); zoomed image of the T cell zone. Contact scores between functional Tfr TIL (CD4+Foxp3+ICOS+) and Tfh TIL (CD4+PD-1-) or functional Tfh TIL (CD4+ $\left.{ }^{+} \mathrm{PD}-1^{+}\right)$or Tfr TIL (CD4+Foxp3 $\left.{ }^{+} \mathrm{COS}^{-}\right)$or TIL-B (CD2O+) in the T cell zone. Magnification: $\times 80$ (upper right); $\times 100$ (upper left); $\times 200$ (lower panels). (F) Using the same mIHC-stained sections as in $\mathbf{E}$, the ratios between (left) total Tfh TIL (PD-1-+PD-1+) and total Tfr TIL (ICOS $\left.+1^{-} \mathrm{ICOS}^{+}\right)$and (right) functional Tfh TIL and functional Tfr TIL are shown for the T cell zones of $5 \mathrm{CC}^{+}$and 3 CC- TLS (TNBC; Student's $t$ test). Statistical testing for all correlations used linear regression analysis. ${ }^{*} P<0.05$.

of the TCF7, CD62L (SELL), and IL7R genes, which is characteristic of effector/memory $\mathrm{T}$ cells (31). In contrast, CD8 $8^{+} \mathrm{PD}-1^{\text {hi }} \mathrm{TIL}$ expressed the highest levels of FASL (FAS ligand), GZMB (granzyme B), and IFNG, genes consistently associated with cytotoxic activity. The immune-checkpoint genes TIM3 (HAVCR2) and CTLA4 together with CD39 (ENTPD1) and CD103 (ITGAE), 2 genes whose coexpression characterizes tumor-reactive CD8 ${ }^{+} \mathrm{TIL}$ (30), were also highly expressed by the PD- $1^{\text {hi }}$ subpopulation. CXCL13-producing $\mathrm{CD}^{+}$TIL were primarily PD- $1^{\text {hi }}$, but divided between $\mathrm{CXCR}^{-}$and $\mathrm{CXCR}^{+}$(Figure 5C), and were principally detected in TN BC (data not shown). Interestingly, CD ${ }^{+}$PD $-1^{\text {hi }}$ TIL were significantly correlated with functional Tfh TIL (Figure 5D) and TfhX13 TIL (Supplemental Figure 5A) in all BC subtypes except luminal A.

Interactions between TIL subpopulations, characterized by mIHC (Figure 5E and Supplemental Figure 5B), confirmed that in addition to activity in the GC (zoom GC), functional Tfh TIL $\left(\mathrm{CD} 4^{+} \mathrm{PD}-1^{+}\right)$colocalize with $\mathrm{CD} 8^{+}$TIL (and occasionally with CD8 ${ }^{+} \mathrm{PD}-1^{+} \mathrm{TIL}$ ) in the $\mathrm{T}$ cell zone (zoom 1; additional clusters are visible in Figure $5 \mathrm{~F}$ ). While $\mathrm{CD} 4^{+} \mathrm{PD}-1^{+} \mathrm{Tfh}$ TIL were relatively numerous, CD8 ${ }^{+} \mathrm{PD}-1^{+}$TIL were more sporadically distributed. $\mathrm{CD}^{+} \mathrm{GZMB}^{+} \mathrm{TIL}$ were also detected in TLS areas containing proliferating tumor cells (based on $\mathrm{Ki}-67^{+}$plus morphology; Figure $5 \mathrm{E}$, zoom 2) together with other $\mathrm{GZMB}^{+} \mathrm{TIL}$ (predominantly CD68 Figure 5G and Supplemental Figure 5B). Interestingly, in a GCnegative TLS, many CD8 ${ }^{+} \mathrm{GZMB}^{+}$TIL were found in the adjacent tumor bed (Supplemental Figure 5C).

The $\mathrm{T}$ cell zones of individual TLS were scored for PD- $\mathbf{1}^{+}$and $\mathrm{GZMB}^{+}$TIL using cIHC-stained consecutive sections (as in Figure 4B), revealing a significant correlation between the prevalence of PD- $1^{+}$TIL (primarily functional Tfh TIL) and $\mathrm{GZMB}^{+}$cells $\left(\mathrm{CD} 8^{+}\right.$ and $\mathrm{CD} 68^{+} \mathrm{TIL}$ ) (Figure $5 \mathrm{H}$ ). Further examination of TIL positioning in the TME detected numerous interactions between $\mathrm{CD}^{+}$and $\mathrm{CD}^{+}$TIL in TLS (Figure $5 \mathrm{~F}$ ) and further characterized the Tfh/Tfr phenotypes of $\mathrm{CD}^{+}$TIL. mIHC also confirmed that TIL in TLS were principally CXCR5 ${ }^{+}$(Supplemental Figure 5D), with multiple functional Tfh TIL in direct contact with $\mathrm{CD} 8^{+} \mathrm{TIL}$ (PD-1 $1^{ \pm}$). Further, in the T cell zone, some Tfr TIL were clearly interacting with functional Tfh and CD8 ${ }^{+}$TIL. Overall, these observations suggest that $\mathrm{CD}^{+}$TIL activation may be regulated in a Tfh-dependent manner.

CD8 ${ }^{+}$PD- $1^{\text {hi }}$ TIL express the canonical Th1 gene IFNG, while CD ${ }^{+} \mathrm{PD}-1^{\mathrm{l} / \mathrm{int}} \mathrm{TIL}$ have expression patterns suggestive of effector memory cells (Figure 5B). To further explore this, we determined the PD- $1^{\text {lo }} / \mathrm{PD}-1^{\text {int }}$ CD8 ${ }^{+}$TIL ratio (Figure $5 \mathrm{~A}$ ) in both the CXCR5 ${ }^{-}$ and $\mathrm{CXCR}^{+}{ }^{\text {ICOS }}{ }^{\text {lo }}$ TIL subpopulations (Supplemental Figure 5E). These analyses detected a low $\mathrm{PD}-1^{\mathrm{lo}} / \mathrm{PD}-1^{\text {int }}$ ratio in all $\mathrm{BC}$ subtypes (particularly TN) compared with tonsils and BC patient blood (Supplemental Figure 5F). Moreover, it was lower in $\mathrm{CXCR}^{+}$compared with CXCR5 ${ }^{-} \mathrm{CD}^{+}$TIL from luminal B and TN BC. The PD- $1^{\mathrm{lo}}$ / $\mathrm{PD}-1^{\text {int }}$ ratio in $\mathrm{CD} 8^{+} \mathrm{CXCR} 5^{+}$TIL was correlated with the functional Tfh/Tfr TIL ratio (Figure 5I) and to a lesser extent with the density of functional Tfh TIL in TN BC (Supplemental Figure 5G). It was also correlated with an increased $\mathrm{PD}-1^{\mathrm{lo}} / \mathrm{PD}-1^{\text {int }}$ ratio in $\mathrm{CD} 8^{+} \mathrm{CXCR} 5^{-}$ TIL, suggesting that in the TME (and/or TLS) when all the active players are present, adaptive immune responses are heightened. This idea is strengthened by the correlation between the functional $\mathrm{Tfh} / \mathrm{Tfr}$ TIL ratio (but not functional Tfh TIL density) and inflammatory cytokines (TNF- $\alpha$, IL-6, and IL-22) in tumor supernatants from primary TN BC (Supplemental Figure $5 \mathrm{H}$ ). These data support a hypothesis that the functional $\mathrm{Tfh} /$ functional Tfr ratio within a given TLS governs its activities and influence on the proximate TME.

Active TLS signal functional immunity and are associated with a good prognosis. In a recent study, we characterized BC TLS with or without GC and linked GC-positive TLS with functional activities (9). Here, we used the NanoString immunology array to comparatively analyze gene-expression patterns from TIL-positive tumors that were scored using dual CD3/CD20 and PD-1/Ki-67 cIHC to separate them into 3 groups: (a) no TLS, but comparable TIL, (b) inactive TLS only, lacking Ki- $67^{+}$and PD-1+ TIL in the B cell follicle and $\mathrm{PD}-1^{+} \mathrm{GZMB}^{+} \mathrm{TIL}$ in the $\mathrm{T}$ cell zone, and (c) a majority of active TLS, the GC distinguished by $\mathrm{Ki}-67^{+}$and $\mathrm{PD}-1^{+}$TIL in the $\mathrm{B}$ cell follicle and/or PD- $1^{+}$and $\mathrm{GZMB}^{+} \mathrm{TIL}$ in the $\mathrm{T}$ cell zone (Figure 6A and Supplemental Table 7).

These data revealed a clear pattern of upregulated immune activation genes specifically associated with tumors containing active TLS. Significantly higher expression of the Tfh genes, CXCL13, CXCR5, IL21, IL21R, ICOS, PD-1 (PDCD1), PD-L1 (CD274), and $C D 38$ together with IFNG, characterized BC with active TLS versus no TLS and reiterated the Th1 orientation of active TLS (Figure 6A). The expression of some immune genes (e.g., ICOS, PD-1, and CD38) was equally low in no TLS/inactive TLS tumors, suggesting that their specific upregulation in active TLS reflects functionality.

The expression of genes associated with $\mathrm{CD}^{+} \mathrm{T}$ cells and their cytotoxic functions, including CD8A, IFNG, GZMB, GZMs (= the mean of GZMA, GZMH, GZMK and GZMM), PRF1, SELL, $I L 7 R$, and IL-12 pathway genes, again produced a pattern of higher expression in active TLS compared with no TLS/inactive TLS tumors. Similar trends were also observed for genes associated with some antigen-presentation markers (LAMP3, CD80, various HLA molecules), immunoregulation (FOXP3, CTLA4, IDO), Th1 pathway transcription factors (TBX21, EOMES, TCF7, STAT1, 
A

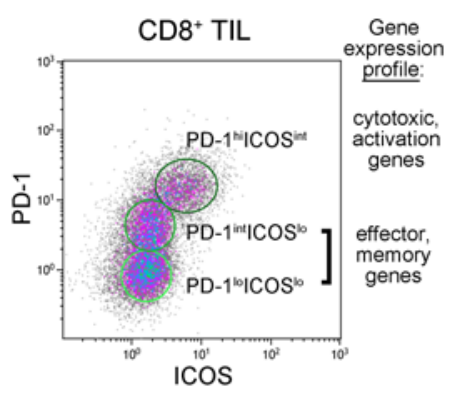

B

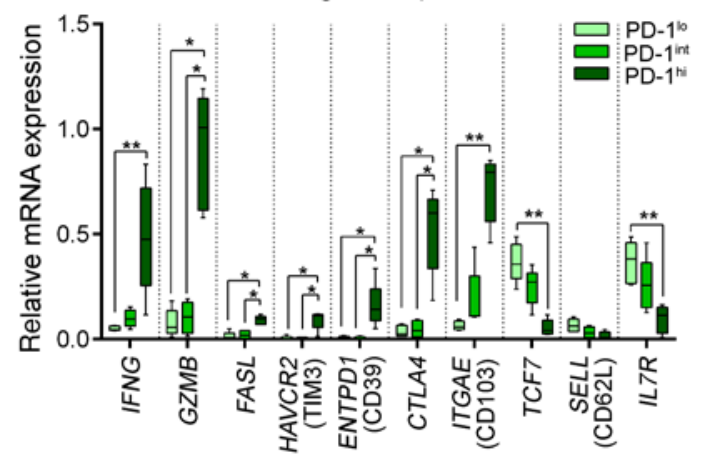

C

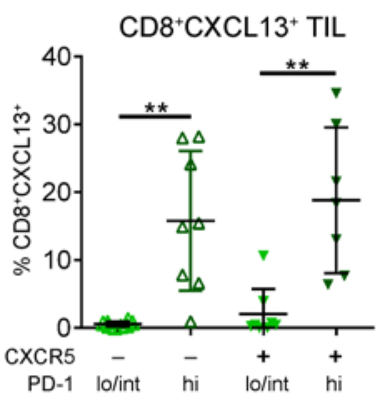

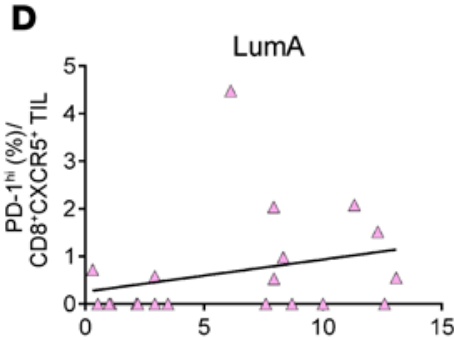
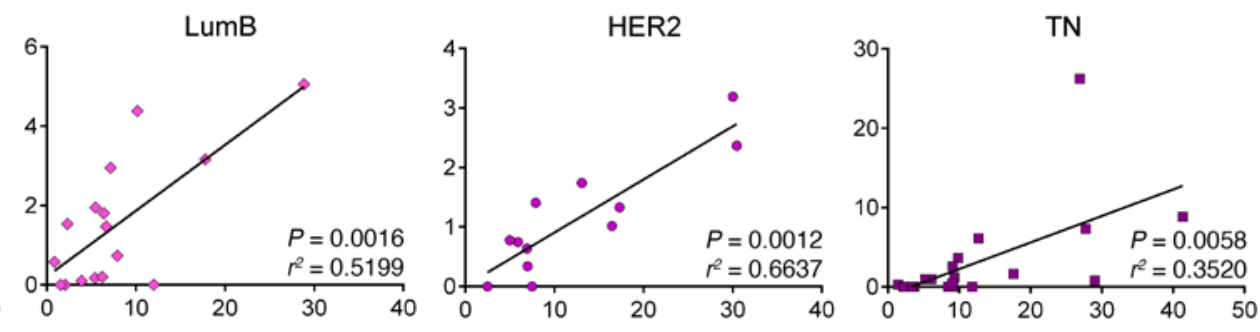

Functional Tfh TIL (\%)/Total Tfh TIL

$\mathbf{E}$

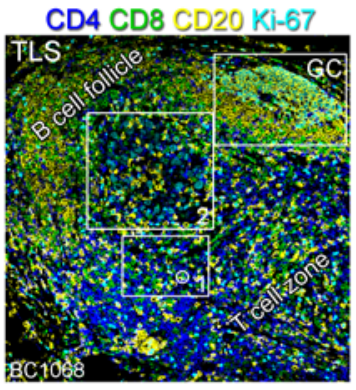

CD4 CD8 PD-1

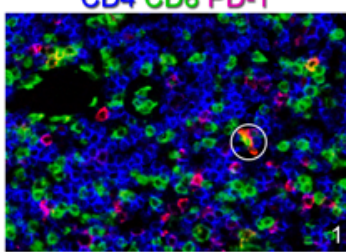

$\mathbf{F}$

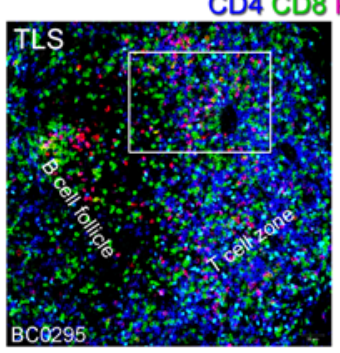

G

CD4 PD-1 CD20 Ki-67

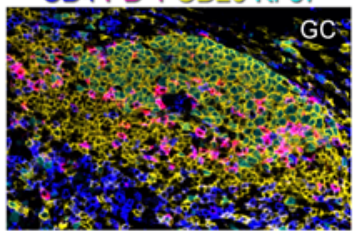

CD8 GZMB Ki-67

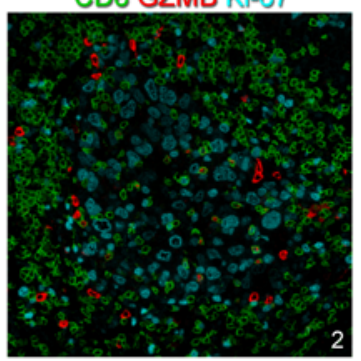

CD68 GZMB

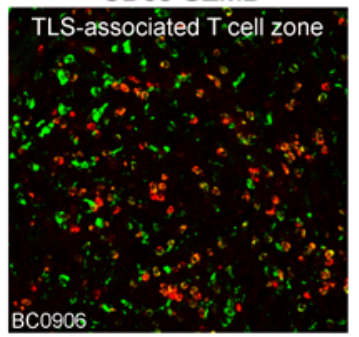

H

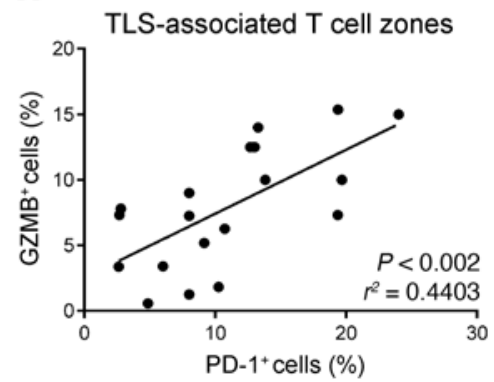

I

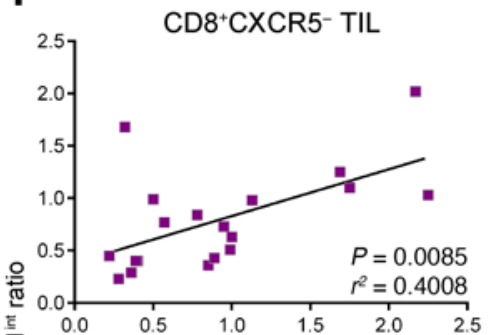

官

定

ì

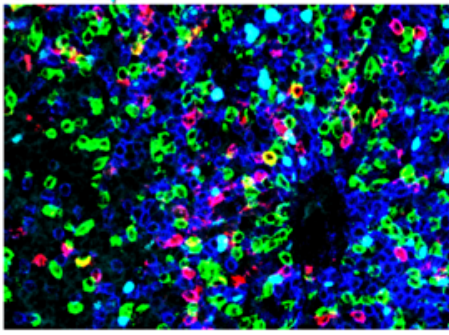

$\mathrm{CD}^{+} \mathrm{CXCR}^{+} \mathrm{TIL}$

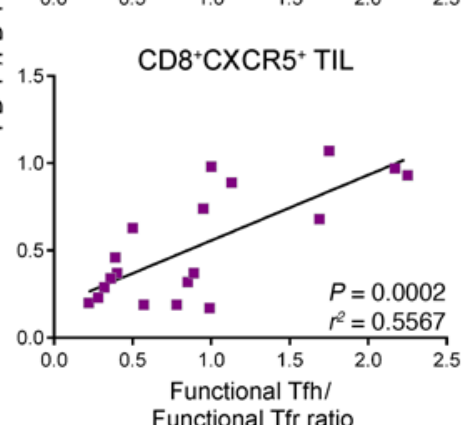


Figure 5. The balance between functional Tfh and Tfr TIL mediates CD8 immune responses in human BC. (A) ICOS and PD-1 on CD8 ${ }^{+}$CD25- TIL (flow cytometry). (B) Gene expression (qRT-PCR) in sorted subpopulations from A ( $n=5$; 1-way ANOVA). (C) Intracytoplasmic CXCL13 (\% positive cells) in subpopulations from $\mathbf{A}(n=8$; 1-way ANOVA). (D) Correlation between the frequencies of functional Tfh (in total Tfh) and activated PD-1 $1^{\text {hil }}$ COS ${ }^{\text {int }}$ (in total CD8 ${ }^{+}$CXCR5 ${ }^{+}$TIL) $(n=71$; LumA [ $n=20]$, LumB [ $\left.n=19\right]$, HER2 ${ }^{+}[n=12]$, TN [ $n=20]$ ). (E) Upper left: mIHC: CD4 (blue), CD8 (green), CD20 (yellow), Ki-67 (cyan), PD-1 (red), CZMB (red) in a TLS (representative BC); upper right: GC zoom, lower left, zoom 1: CD4+PD-1+ TIL (surrogate for functional Tfh TIL; pink) and CD8 $8^{+} \mathrm{PD}-1^{+}$TIL (surrogate for activated CD8 ${ }^{+}$TIL; yellow) in the T cell zone and lower right, zoom 2: $\mathrm{CD}^{+} \mathrm{CZMB}^{+} \mathrm{TIL}$ (green membrane/red cytoplasm), CD4CD8-CD20-CZMB+ TIL (red sub-membrane) and tumor cells (cyan nucleus). Magnification: upper left $\times 50$; upper right $\times 125$; lower left $\times 200$; and lower right ×135. (F) mIHC: CD4 (blue), CD8 (green), PD-1 (red), and Foxp3 (cyan). Left: TLS T cell zone and B cell follicle (representative BC, TLS in Supplemental Figure 5D); right: zoomed image of TLS T:B border showing functional Tfh TIL (pink membrane), activated CD8+PD-1+ ${ }^{+}$TL (yellow membrane), and Foxp $3^{+} \mathrm{CD} 4^{+} \mathrm{Tfr}$ TIL (cyan nucleus). Magnification: left $\times 80$; right $\times 250$. (C) $\mathrm{mIHC}$ : CD68 (green) and CZMB (red) in TLS-associated T cell zone; $\mathrm{CD68}^{+} \mathrm{CZMB}^{+}$TIL (orange); CD68- $\mathrm{CZMB}^{+}$TIL (red; considered CD8 ${ }^{+}$). Magnification: $\times 100$. (H) PD- $1^{+}$cells (functional Tfh TIL) and GZMB ${ }^{+}$cell (CD8 ${ }^{+}$and CD68 ${ }^{+}$TIL) frequencies in TLS-associated T cell zones ( $n=76$ TLS; 20 tumors) correlated with CD3/CD2O, PD-1/Ki-67, and GZMB clHC scores (consecutive sections; each point = mean TLS/tumor; same tumors as in Figure 4C). (I) Ratio between functional Tfh and Tfr TIL correlated with the ratio between PD-1 $1^{10}$ and PD-1int $T$ cells (flow cytometry); upper panel: CD8 ${ }^{+}$CXCR5 ${ }^{-}$TIL, lower panel: CD8 ${ }^{+}$CXCR5 ${ }^{+}$TIL (TN BC; $n=18$ ). Statistical tests used linear regression analysis for all correlations. ${ }^{*} P<0.05$; ${ }^{* *} P<0.01$.

STAT4, NFATC2), B cell differentiation (POU2AF1, PAX5), and innate immunity $(R U N X 3)$, all important players in regulating immune activation. The close relationship between the presence of functional Tfh, activated CD8 ${ }^{+}$TIL, and Tfr TIL together with high expression of key immune genes suggests that $\mathrm{BC}$ containing a majority of active TLS has tipped the balance to favor effector over regulatory activities. This results in the generation of critical antitumor immune responses directly at the tumor site.

We next scored all active TLS and inactive TLS (defined above, exemplified in Supplemental Figure 6) in individual HER2 ${ }^{+}$and TN tumors from our prospective BC cohort $(n=48$; $>3$ years of follow-up; Supplemental Table 8). Disease-free survival analysis comparing TLS densities in tumors from patients with more than 1 $\mathrm{TLS} / \mathrm{cm}^{2}$ to those with 1 or fewer TLS $/ \mathrm{cm}^{2}$ did not reveal significant differences (Figure 6B). However, the analysis of only tumors with a minimum density of $1 \mathrm{TLS}\left(\right.$ per $\left.\mathrm{cm}^{2}\right)$ divided into more than 2 active TLS versus 2 or fewer active TLS revealed that all patients in the more than 2 active TLS group were disease free from 36-86 months (variation due to postsurgery follow-up), except for $1 \mathrm{TN}$ patient whose tumor contained 3 of 17 weakly active and 14 of 17 inactive TLS. Interestingly, disease-free patients had overall TLS densities similar to those who relapsed, but notably, the former had higher densities of active TLS (Figure 6C). MIHC examination of functional TIL immunophenotypes in TLS confirmed that functional Tfh TIL, proliferating TIL-B, and lower Tfr TIL were associated with a lack of recurrence (Figure 6D). Further, a higher ratio of functional Tht to functional Tfr was associated with disease-free survival, while the total $\mathrm{Tfh} / \mathrm{Tfr}$ ratio was not. Overall, these data demonstrate the importance that active TLS play in guiding immune responsiveness in the TME.

\section{Discussion}

The generation of immune responses at the tumor site, currently quantified by the morphological evaluation of stromal TIL (2), has been linked with positive clinical outcomes in many solid tumor types, including BC $(32,33)$. Cumulative evidence supports the idea that specific cellular interactions and soluble factors, reflecting effective antitumor immune responses, come together when TIL are organized in TLS $(5,6)$. Three key gene-expression studies published last year highlighted the importance of TLS and TIL-B in predicting responses to immunotherapy and survival in patients with sarcoma (12), melanoma (10, 11), and renal cell carcinoma (11). TIL-B have also been associated with humoral immune responses (34) and positive clinical outcomes in untreated cancer patients $(23,35)$.

Closely linked with TIL-B functionality are their primary helpers, Tfh cells, which have increasingly been associated with TLS and/or antitumor immune responses in a variety of tumor types. Gene-expression analysis of TIL subpopulations in colorectal carcinoma identified a central role for Tfh TIL and TIL-B in antitumor immunity (22). In patients with hepatocellular carcinoma, a reduction in circulating and/or tumor-associated Tfh cells was correlated with disease progression and reduced disease-free survival (36). Tfh TIL were also positively correlated with tumor mutational burden in lung adenocarcinoma (37). Tfh cells in the blood and pulmonary metastasis of patients with differentiated thyroid cancer were recently shown to be higher in patients with metastatic disease; however, these cells produced lower quantities of IL-10, IL-21, and CXCL13, suggesting reduced helper functions could participate in disease progression (38). Our BC studies, expanded by the data presented here, strongly link functional Th1-oriented Tfh TIL with TIL-B, CD8 ${ }^{+}$TIL, fewer Tfr cells, TLS, and better disease outcomes and suggest these specialized TIL are an important target of PD-1/ PD-L1 immunotherapy $(3,4,14,23,39,40)$.

Much has been learned from studies of Tfh cells infiltrating tissues and circulating in the blood of patients with nonmalignant human diseases, where they are associated either with pathogenesis (e.g., autoimmunity) or resolution/control (e.g., viral infection). Some HIV patients on antiretroviral therapy have virus-specific circulating Th1-oriented Tfh cells, whose expansion is thought to be driven by persistent viral antigen (41). HIV-1 controllers, characterized by low viral loads, produce broadly neutralizing antibodies that parallel a high frequency of circulating Tfh cells (42). A recent study of patients infected with SARS-CoV-2 (COVID-19) detected higher numbers of circulating virus-specific Tfh cells and neutralizing antibodies in individuals recovering from severe compared with nonsevere disease (43). Additionally, postmortem analyses of patients who succumbed to COVID-19 found a lack of GC and Tfh cells, suggesting the virus may have destroyed their ability to mount neutralizing antibody responses (44). Overall, the presence of increased circulating Tfh cells during viral infection is thought to reflect Tfh-mediated B cell differentiation and maturation in the GC, which promotes virus-specific neutralizing antibody production and memory cell generation. In autoimmune diseases, via the same mechanisms, Tfh cells promote destructive activities, exemplified by increased disease pathogenesis in RA patients who accumulate Th1-oriented Tfh cells in their synovial fluid (45).

A specialized CXCR5 ${ }^{-}$CXCL13-producing $\mathrm{CD}^{+}{ }^{+}$Tfh subpopulation discovered in RA (Tph) (16) and BC (TfhX13) $(14,17)$ has more 
A
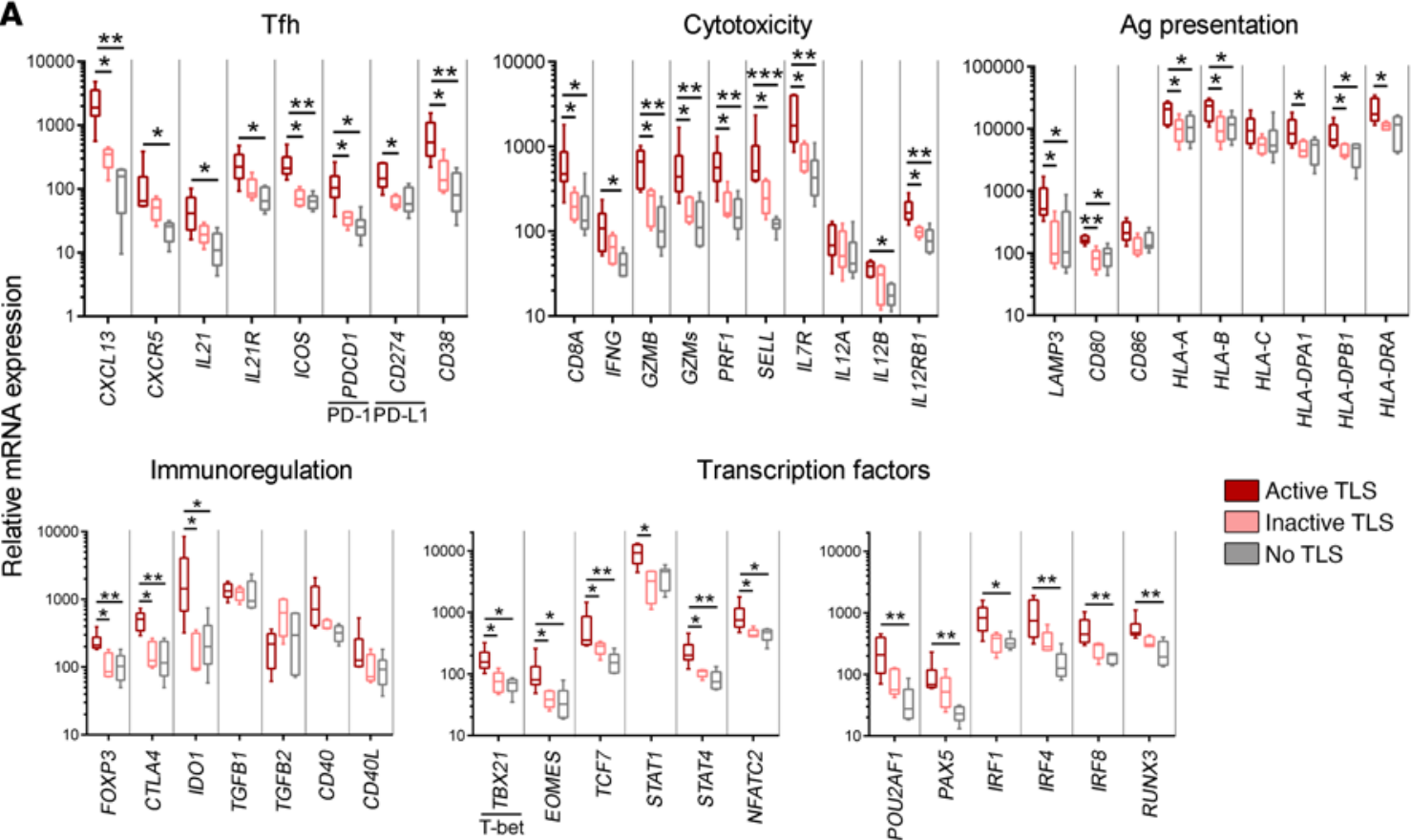

\section{on factors}

Active TLS

Inactive TLS

$\square$ No TLS

B

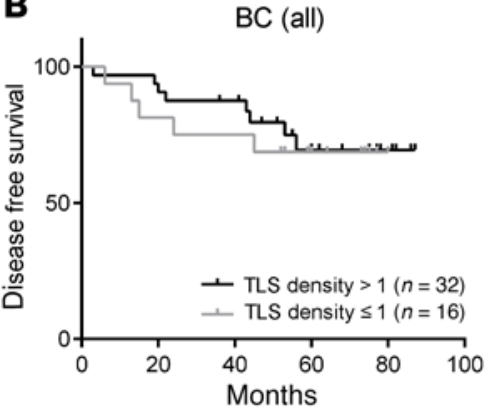

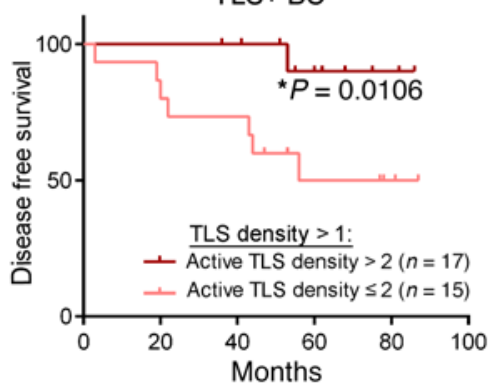

TIL compostion of TLS in BC

D

Functional Tfh TIL

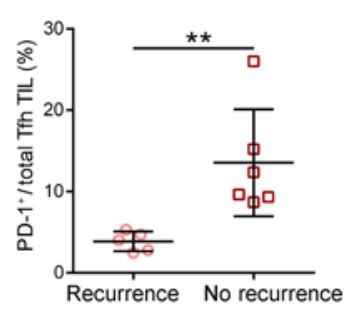

Tfh TIL to Tfr TIL

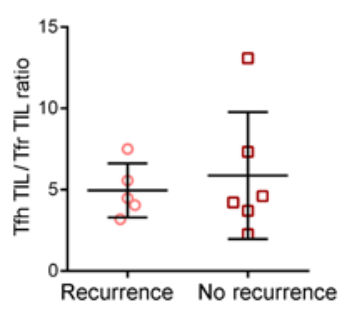

Proliferating TIL-B

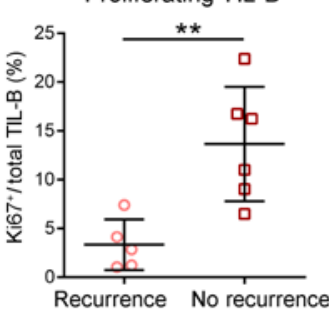

Functional Tfh TIL to

Functional Tfr TIL

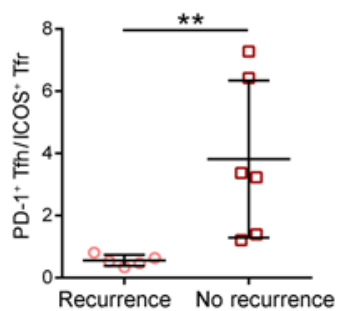

TLS+ BC

Functional Tfr TIL
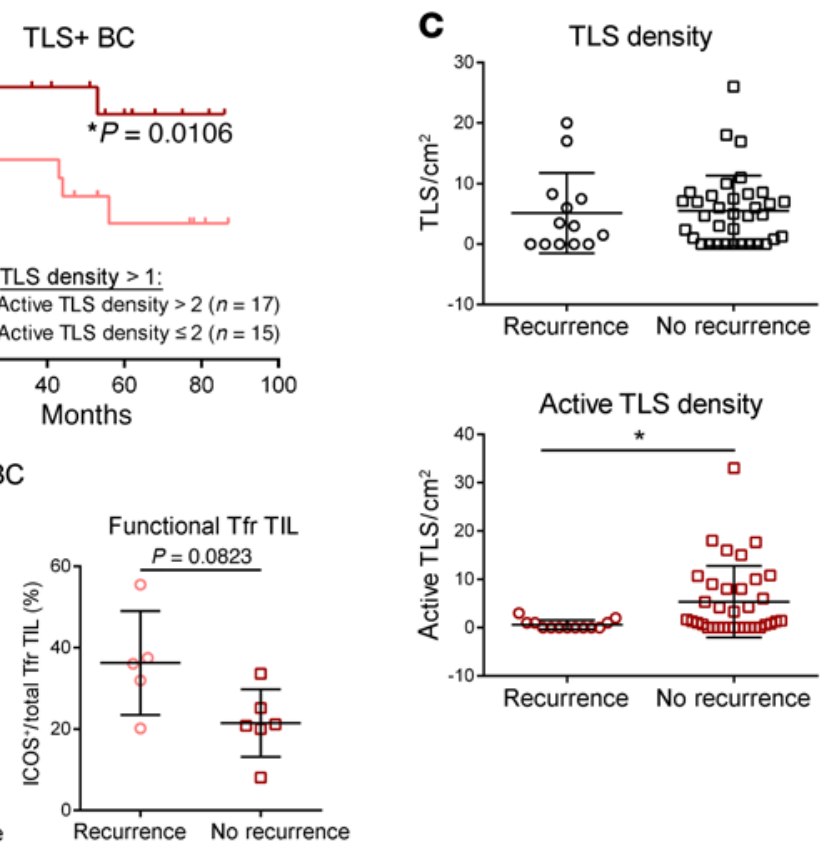
Figure 6. Active TLS signal adaptive immunity and are associated with a good prognosis. (A) Gene-expression analysis using the Nanostring Pan Cancer Immune Profiling Panel on total tumor RNA extracted from FFPE sections of highly infiltrated $\mathrm{BC}$ tumors (TN and HER2 ${ }^{+}$). The tumors are divided into active TLS (PD-1+Ki-67+/-, $n=6$ ), inactive TLS (PD-1-Ki-67-, $n=$ 5), and TIL without TLS (no TLS, $n=6$; 1-way ANOVA). TLS numbers and activities were scored on dual cIHC-stained (CD3/CD2O and PD-1/Ki-67) consecutive sections that followed those for RNA extraction. (B) Kaplan-Meier curves for disease-free survival. Left: relative to TLS density ( $n=48 ; \mathrm{HER2}^{+}$ and TN BC); right: relative to active TLS density $\left(n=32 ; \mathrm{TLS}^{+} \mathrm{BC}\right.$ only) in the resected tumor. (C) Upper graph: total TLS densities; lower graph: active TLS densities compared between BC patients with $(n=13)$ or without $(n=$ 35) disease recurrence (Student's $t$ test). (D) TIL subpopulations in all TLS scored on mIHC tissue sections (stained as in Figure 4E) from 11 BC (5 with and 6 without recurrence). Each point represents the mean of all TLS in a given tumor (Student's $t$ test). Disease-free survival based on (upper left) frequency of functional cells (PD-1+) in total Tfh TIL, (upper middle) proliferating cells (Ki-67+) in TIL-B, and (upper right) functional cells (ICOS+) in $\mathrm{Tfr}$ TIL. Ratios between (lower left) total Tfh TIL (PD-1- + PD-1+) and total Tfr TIL $\left(\mathrm{ICOS}^{-}+\mathrm{ICOS}^{+}\right.$) and (lower right) functional Tfh TIL and functional Tfr TIL relative to disease-free survival. ${ }^{*} P<0.05 ;{ }^{* *} P<0.01$; ${ }^{* *} P<0.001$.

recently been associated with disease severity in systemic lupus erythematosus and other autoimmune diseases (46). A characteristic of TfhX13 TIL and Tph in RA is their high expression of the IL21 and IFNG genes (17). IL-21, a pleiotropic cytokine acting on immune and other cells, promotes B cell differentiation, Tfh cell development, and $\mathrm{CD} 8^{+} \mathrm{T}$ cell survival, thereby driving antitumor or autoreactive immune responses. IFN is an important multifunctional cytokine well known for directing immune responses to pathogens and tumors as well as its involvement in autoimmune pathologies, with Th1-mediated immunity at the forefront of these activities. IL-21 and IFN- $\gamma$ production by Tph cells is linked with exacerbated production of autoantibodies, while TfhX13 recruitment of functional $\mathrm{CXCR}^{+}{ }^{+}$Tfh TIL, CD8 ${ }^{+}$TIL, and TIL-B to TLS promotes antitumor effector memory TIL maturation and is associated with survival. The higher expression of IL21 and IFNG detected in BC with active TLS compared with no/inactive TLS reinforces the notion that key Th1-driven and humoral responses are generated when active structures are resident in the TME.

Analysis of CXCR5 ${ }^{-}$and $\mathrm{CXCR}^{+} \mathrm{CD}^{+} \mathrm{PD}-\mathrm{1}^{\text {hi }}$ TIL revealed both subpopulations express IFN and GZMB, with most also making CXCL13. Our early studies detected CXCL13-producing CD8 ${ }^{+}$ TIL in BC, but at lower frequencies than TfhX13 TIL (14). These TIL were subsequently described as a more significant subpopulation in other solid tumors, including lung cancer, in which $\mathrm{CD}^{+} \mathrm{CX}-$ CL13 ${ }^{+} \mathrm{PD}-1^{\text {hi }}$ TIL were identified as the most tumor-reactive TIL subpopulation (18). In Hodgkin lymphoma, CD8 ${ }^{+} \mathrm{CXCR}^{+} \mathrm{ICOS}^{+}$ $\mathrm{T}$ cells have gene-expression patterns similar to those of IL-4-, IL-21-, and CXCL13-producing CD4+ $4^{+}$fh (47). In an ovarian cancer study (19), TGF- $\beta$-dependent CD8 ${ }^{+} \mathrm{CD} 103^{+} \mathrm{CXCL} 13^{+} \mathrm{TIL}$ were correlated with TIL-B recruitment and TLS formation, similarly to TfhX13 in BC $(4,14)$. Markers of CD $8^{+} \mathrm{CD} 103^{+}{ }^{+}{ }^{2} C L 13^{+}$TIL have also been correlated with survival, suggesting they may synergize with TfhX13 TIL to induce CXCL13-dependent Th1-oriented TLS formation and immune responses. CXCL13-directed migration of $\mathrm{CXCR}^{+}$TIL to BC-associated TLS could thereby promote functional and/or suppressive interactions based on the relative balance and frequency of each subpopulation.
Studies of TLS in cancer and other chronic inflammatory diseases have revealed that these structures can vary in activity. In 2014, 2 types of TLS were described in squamous cell carcinomas: (a) classical TLS as B cell aggregates with follicular dendritic cell (FDC) networks and GC B cells and (b) nonclassical TLS as B cell aggregates with unstructured FDC accumulations that lack or have few GC B cells (48). A 2016 ovarian cancer study identified 4 types of lymphoid aggregates, classifying the type III aggregates as mature TLS with ongoing GC reactions (8). In 2018, 3 developmental stages of TLS were distinguished in a murine model of lung TLS formation, including (a) early TLS characterized by lymphoid aggregates without FDC, (b) primary follicle-like TLS containing FDC but lacking a GC, and (c) secondary follicle-like TLS with a GC (7). Our recent work identified the transcription factor Foxp1 as a marker for GC-positive (Foxp1 ${ }^{-}$) and GC-negative (Foxp1 ${ }^{+}$) TLS and demonstrated that the colocalization of $\mathrm{Ig}^{+} \mathrm{Ki}-67^{+}$TIL-B differentiation in tight correlation with $\mathrm{CD} 4^{+} \mathrm{PD}-1^{+}$Tfh TIL in the GC signals an active TLS (9).

Building on previous work by us and others, the data presented here show that active TLS are linked with antitumor immunity as a consequence of close interactions between functional Th1-oriented $\mathrm{CXCR}^{+} \mathrm{Tfh}$ TIL and other CXCR5 ${ }^{+}$TIL. Our demonstration that functional Tfh TIL produce IFN- $\gamma$ and can stimulate B cell differentiation ex vivo contrasts with studies of healthy donor tonsillar Tfh cells, which only promote the latter. Functional Tfh TIL drove the maturation and differentiation of naive CXCR5 ${ }^{+}$TIL-B to memory cells and antibody-secreting cells in our ex vivo assay. These data also confirmed the robust link between Tfh TIL and GC TIL-B previously detected by flow cytometry $(14,23)$, supporting the conclusion that interactions between functional Tfh TIL and GC TIL-B in a TLS are required for Ig production and memory cell generation.

$\mathrm{CD}^{+}$TIL activation in a TLS could also be regulated in a Tfhdependent manner. This scenario would include TfhX13 TILmediated recruitment of $\mathrm{CD} 8^{+} \mathrm{CXCR} 5^{+} \mathrm{TIL}$ to TLS, triggering their interactions with functional Tfh TIL and expanding Th1-mediated immune responses. In addition to functional Tfh cells promoting GC B cell maturation and Ig production, they could also trigger chronically activated CD $8^{+}$TIL to reexpress IFN- $\gamma$ and GZMB. This would promote (re-)activation of $\mathrm{CD} 8^{+} \mathrm{PD}-1^{10 / \text { int }} \mathrm{TIL}$ and migration of GZMB ${ }^{+}$TIL out of the TLS. While these data on Th1-oriented Tfh-infiltrating tumors are new, they are supported by the neoantigen reactivity of CD $8^{+} \mathrm{PD}-1^{\mathrm{hi}} \mathrm{TIL}$ in lung cancer (49) and the association of $\mathrm{CD}^{+}{ }^{+} \mathrm{ICOS}^{+} \mathrm{TIL}$ with a Th1 effector phenotype, higher PD-1 expression, and improved survival in colorectal cancer (50). Expansion of Th1-biased Tfh cells has also been observed in lymph nodes from chronically HIV-infected individuals (51). Recent studies identify Th1-oriented $\mathrm{CD}^{+}{ }^{+} \mathrm{CXCR} 5^{+} \mathrm{T}$ cells as important emerging players in infection and cancer, with initial data suggesting they function as "nonexhausted" enhanced memory effectors (TCF7 dependent) when activated in chronic inflammatory microenvironments (52). As chronically activated effector cells, these specialized CD $8^{+}$TIL could be a reflection of their TLS activities rather than an exhausted phenotype, a concept that requires further investigation. Overall, these data suggest that the role of functional Tfh TIL in tumor-associated TLS is to orchestrate both adaptive and humoral immune responses.

TLS immune cell activities are controlled by specialized regulatory cells, including the well-known Tregs and their more recently identified follicular counterparts, Tfr cells. We determined that functional 
Tfr TIL are similar to Tfr in human tonsils, both identified here by their demethylated FOXP3 gene and high expression of GARP, a receptor that binds and activates latent TGF- $\beta$. Our data show that GARP ${ }^{+}$TIL not only reflect the presence of activated Tregs, but importantly, also functional Tfr TIL in human BC. A human hepatocellular carcinoma study found Foxp ${ }^{+} \mathrm{GARP}^{+}$Tregs in the blood were correlated with impaired immune responses in patients (53). In human ovarian cancer, GARP was 1 of 8 markers used to demonstrate higher frequencies of Th17 cells transitioning to Tregs in the ascities (54). A murine colon cancer model study has more recently shown that GARP can regulate Treg activity and thereby $\mathrm{CD}^{+}$and $\mathrm{CD} 8^{+}$activation (55). Our data provide important information by showing that the relative abundance of GARP ${ }^{+}$Tfr TIL, rather than GARP ${ }^{+}$Treg TIL, directly influences the level of Tfh TIL helper activity. Further, we show that TGF- $\beta$ plays a role in this process, likely via high GARP expression on functional Tfr TIL. These data suggest that TIL entering the T cell zone are carefully monitored and selected for access to the B cell follicle, where they are further controlled for maturation and differentiation, which is similar to observations in human mesenteric lymph nodes (28). These close interactions highlight the importance Tfr TIL play in controlling Tfh TIL-dependent TIL-B differentiation in the TLS. We hypothesize that the balance between functional Tfh and functional Tfr TIL dictates the activity of a TLS and thereby reflects the level of immune activation/suppression within a given tumor. Tfr TIL may constitute a new focus for immunotherapy, potentially via targeting IL-35 expression, specific for TIL relative to tonsillar T cells with higher expression on Tfr compared with Treg TIL.

Tfh and Tfr TIL functionality was correlated here with $\mathrm{CD} 4^{+} \mathrm{CX}-$ $\mathrm{CR}^{+}$subpopulations expressing varying intensities of surface PD-1 and ICOS, both receptors associated with signals that instruct $\mathrm{T}$ cell differentiation and function (15). Important signals initiate from ICOS/ICOSL interactions, a pathway known to regulate Tfh differentiation and stimulate CXCR5 and Bcl6 upregulation. Activation of this pathway triggers migration of early $\mathrm{Tfh}$ cells to the $\mathrm{B}$ cell follicle, first to the T-B border then the GC, where $\mathrm{ICOS}^{+} \mathrm{Tfh}$ interactions with $\mathrm{ICOSL}^{+} \mathrm{B}$ cells are a prerequisite for successful GC reactions. ICOS is a critical regulator of Tfh, with ICOS-deficient humans characterized by severe defects in humoral immunity and immunodeficiencies associated with reduced Tfh (56). In contrast, increases in peripheral $\mathrm{ICOS}^{+} \mathrm{Tfh}$ in healthy donors receiving a hepatitis $B$ vaccine were positively correlated with their antibody responses (57). In cancer, the intensity of ICOS/ICOSL expression and activity dictates pro- or antitumor immune pathway functionalities (58), with our data suggesting that Tfh and Tfr could compete for ICOSL, making them potentially relevant targets for ICOS blockade.

PD-1, initially identified for its ability to attenuate TCR and BCR signaling after activation, is now an established immune-checkpoint molecule and principal target of cancer immunotherapy (59). PD-1 surface intensity on Tfh cells guides their expansion, location, and functionality (60). In cancer, interactions between PD-1 ${ }^{+}$and PD-L1 ${ }^{+}$ cells can interfere with immune responses while their therapeutic blocking successfully restores antitumor immunity in some patients. The recent positive association between TLS and TIL-B for disease-free and overall survival in patients following immunotherapy (10-12) is further developed by data from a murine BC model linking $\mathrm{Tfh} / \mathrm{B}$ cell interactions to this response (61). Additionally, dampened $\mathrm{CD}^{+} \mathrm{T}$ cell functions, initially shown in chronic viral infections to be revived by blocking the PD-1/PD-L1 pathway, are now similarly recoverable in cancer patients (59).

TfhX13, functional $\mathrm{Tfh}$, and $\mathrm{CD} 8^{+} \mathrm{PD}-1^{\mathrm{hi}} \mathrm{TIL}$ are all potential targets of anti-PD- 1 treatment. CD ${ }^{+} \mathrm{CXCL} 13^{+} \mathrm{PD}-1^{\text {hi }}$ TIL were previously shown to be deficient in effector cytokine production (18), similar to BC TfhX13 TIL in the Tfh functional assay shown here. Nonetheless, these CXCL13-producing CD8 ${ }^{+}$TIL were predictive for clinical outcome in lung cancer patients treated with anti-PD-1. Further, a Th1-like $\mathrm{CD} 4{ }^{+} \mathrm{CXCL13}{ }^{+} \mathrm{T}$ cell expressing the transcription factor BHLHE4O (also with high expression of PDCD1 [PD-1], ICOS, CXCR3, TIGIT, and HAVCR2 [TIM3]) was preferentially enriched in microsatellite-instable colorectal carcinoma and associated with positive responses to immune checkpoint blockade (62). Based on the recent studies highlighting the importance of TLS and TIL-B in immunotherapy responses $(10-12,61)$, our data suggest that functional Th1-oriented Tfh TIL (CXCR5 ${ }^{+}$PD-1 ${ }^{\text {hi }}$ ) in TLS are a key target of PD-1/PD-L1 blockade. These data provide a rationale for the increases in $\mathrm{B}$ cell differentiation and IFN- $\gamma$ gene expression observed in patients responding to immune checkpoint blockade (11).

The present compositional, positional, and functional analysis of CXCR5 ${ }^{+}$TIL subpopulations recruited to TLS extends our understanding by showing that the prevailing balance between functional Th1-oriented Tfh TIL and functional GARP ${ }^{+}$Tfr TIL can promote or inhibit interactions linked to the generation of active or inactive TLS, respectively. As functional Tfh and Tfr TIL commingle in the TLS, their relative balance controls the extent of active humoral immune responses. These data suggest that when the balance favors functional Tfh over Tfr TIL in an active TLS, Tfh cellular interactions and soluble gene products generate a Th1 microenvironment that influences TIL in the TME. These data support a hypothesis that the functional $\mathrm{Tfh} /$ functional Tfr ratio within a given TLS governs its activities and influence on the proximate TME.

TLS arise at sites of chronic inflammation or infection due to a persistent imbalance between the recruitment and clearance of immune cells in the inflamed tissue. The long-term disease-free survival of some TIL-high patients whose TLS-containing tumors are resected prior to treatment suggests that robust antigen-specific antitumor memory responses are generated, then move into the periphery and help to control residual disease. We identified disease-free patients whose untreated primary tumors contained active TLS, characterized by subpopulations of functional Th1-oriented Tfh TIL and activated Th TIL, CD8 ${ }^{+}$TIL, and TIL-B. The formation of active TLS at the tumor site can be interpreted as helping to position specific cellular interactions necessary for the generation of antigen-specific humoral and cytotoxic immune responses in a microenvironment protected from direct tumor-mediated suppression. This view is consistent with survival studies in numerous cancer types showing that TLS are a favorable biomarker (6), which together with the recent work linking them to immunotherapy responsiveness, highlight their importance in antitumor immunity. Importantly, the specific immune cell subpopulations and gene-expression patterns associated here with active TLS, lacking in tumors with inactive TLS or TIL, but no TLS, are clearly linked with positive clinical outcomes. This distinction between active and inactive TLS suggests that, while driving TIL to and into the tumor is important, TIL instruction by functional Th1-oriented Tfh TIL may be a critical factor in promoting the multifactorial immune responses needed to generate long-lasting 
immunological memory to the tumor that functionally help control residual disease and prevent relapse.

\section{Methods}

For further details, see Supplemental Methods.

Patient samples. Fresh breast tissues (day of surgery), including 168 invasive ductal breast carcinomas (untreated, nonmetastatic) and matched nonadjacent nontumor (NANT) tissues as well as normal breast from 11 patients undergoing mammary reduction (controls), were used in this study. BC patients were treated at the Institut Jules Bordet between August 2015 and January 2018; patient information is in Supplemental Table 1. Fresh tissue samples were measured and weighed and an imprint was H\&E stained for tissue identification. Fresh tonsils and spleens were additional controls. For survival analysis, formalin-fixed, paraffin-embedded (FFPE) tumor blocks were prospectively collected with clinical and survival data from untreated, invasive ductal BC patients who underwent surgery between 2012 and 2017 (Supplemental Table 8); of these, 48 patients with TN $(n=27)$ or HER2 ${ }^{+}\left(n=9\right.$ HER2 ${ }^{+} \mathrm{HR}^{-} ; n=12$ $\mathrm{HER} 2^{+} \mathrm{HR}^{+}$) were selected for survival analysis. All patients were treated in the adjuvant setting using standard-of-care protocols.

TLS scoring. TLS and TIL aggregates were scored on dual CD3/ CD20 cIHC-stained FFPE tissue sections from human BC. TLS are distinguished by TIL organized in a T cell zone or B cell follicle; TIL aggregates are unorganized TIL clusters. TLS were identified on a CD3/CD20 cIHC-stained section and quantified for activity on consecutive PD-1/ Ki-67 and GZMB cIHC-stained consecutive sections.

Tissue homogenates. Fresh surgical tissues (tumors, NANT, tonsils, and spleen) were processed as previously described (29). Lymphocytes from PBMCs, tonsils, and spleens were purified by Lymphoprep density gradient centrifugation and washed $3 \times$ before antibody staining.

Tfh functional assay. Sorted human $\mathrm{CD} 4^{+} \mathrm{TIL}$ or tonsillar $\mathrm{CD} 4^{+} \mathrm{T}$ cells (10,000 cells/well) were activated with staphylococcal enterotoxin B (SEB) $(500 \mathrm{ng} / \mathrm{ml}$; Sigma-Aldrich) together with human splenic B cells $(25,000$ cells/well) in complete RPMI 1640 supplemented with $10 \%$ FBS in a method modified from one previously described (63). For consistency across tumors, B cells were isolated (Human B Cell Isolation Kit II; Miltenyi Biotec) from the same healthy donor spleen for all assays, with $\mathrm{CD} 19^{+}$purity ( $\geq 95 \%$ positive cells) controlled using flow cytometry (Gallios 10/3 cytometer, Beckman Coulter). A well was harvested at day 3 to quantify CD25 and ICOS as markers of activation. Supernatants were harvested at day 7 for Ig and cytokine analysis. Treg and Tfr suppressive activities on tonsillar Tfh cells (ratio 1:1) were tested using sorted tonsillar cells in the assay. An anti-TGF- $\beta 1,-2,-3$ blocking antibody $(10 \mu \mathrm{g} / \mathrm{ml}$; MAB 1835-100, R\&D Systems) or recombinant human TGF- $\beta 1$ (0.05-5 ng/ml; 100-21, Peprotech) was added to some wells.

Gene-expression analysis. Twenty-four untreated primary invasive ductal BCs were selected for analysis. Total RNA was extracted from three $5 \mu \mathrm{m}$ tissue sections using the RNeasy FFPE Extraction Kit (QIAGEN). RNA quantity and quality were analyzed using Qubit and the RNA 6000 Pico Kit (Agilent 2100 Bioanalyzer). RNA meeting the quality control standards was analyzed with the NanoString Pan Cancer Immune Profiling Panel (NanoString Technologies), which includes probes for 770 genes with 109 cell-surface markers capable of quantitating 24 different TIL subpopulations, 30 genes for common CD antigens, more than 500 genes that measure immune responses, including checkpoint regulation/signaling, and 40 Pan Cancer reference genes. Raw data from the run were processed and normalized with standard NanoString protocols using their nSolver Analysis Software. Unsupervised analysis was performed by a biostatistician.

Statistics. $P$ values for 2-group comparisons were generated using a 2-tailed Student's $t$ test, while $P$ values for multiple-group comparisons were generated using a 1-way ANOVA test, either paired or unpaired depending upon the samples. The linear regression method was used for all correlation analyses. $P<0.05$ was considered significant.

Study approval. The present human studies were reviewed and approved by the Medical Ethics Committee of the Institut Jules Bordet (CE 1981, CE2550). BC patients gave informed consent prior to their participation in the study. Other residual tissues were obtained anonymously, without access to clinical data.

\section{Author contributions}

GN, MLF, and KWG conceived the research and designed experiments, and GN and MLF performed the majority of experiments. DL and LC provided patient samples. MLF, NT, and CS collected clinical data. $\mathrm{AB}$ and $\mathrm{CN}$ performed all the chromogenic and multiplex stainings with support from SG. ADW, GGVDE, RS, and DL performed pathological evaluations and scoring of cIHC-stained tissues. GN, MLF, PDS, and EM performed flow cytometry, and GN, MLF, and HD performed cell-sorting experiments. SL provided anti-GARP antibodies as well as scientific and methodological expertise on GARP. $\mathrm{AB}$ and MLF cut and extracted RNA from tissue sections, and HL and KT performed NanoString array experiments and data analysis. KWG supervised the research. GN, MLF, and KWG analyzed and interpreted the data. GN performed statistical analyses. GN and KWG wrote the paper. All authors provided advice on the final manuscript.

\section{Acknowledgments}

The authors thank Pierre Coulie and Pierre van der Bruggen for scientific discussions and Jean Fain and Sophie Pattyn for their experimental help as research interns. Confocal microscopy images were acquired at the Center for Microscopy and Molecular Imaging (CMMI), which is supported by the Walloon Region and the ERDF (Wallonia-Biomed portfolio, 411132-957270). This work was supported by grants from the Fonds de la Recherche Scientifique (FNRS; grant J.0005.20) Opération Télévie (grants 7.4568.17, 7.4559.18, 7.6518.18, and 7.4619.19), Les Amis de l'Institut Bordet (grants 201812 and 2020-20), the Medic Foundation, and Fonds Lambeau-Marteaux. GN and SG are postdocs, and MLF and NT are doctoral fellows of the FNRS-Operation Télévie.

Address correspondence to: Karen Willard-Gallo, Molecular Immunology Laboratory, Institut Jules Bordet, Université Libre de Bruxelles, 127 Blvd. de Waterloo, B1000 Brussels, Belgium. Phone: 32.2.541.3739; Email: karen.willard-gallo@bordet.be.
1. Paijens ST, et al. Tumor-infiltrating lymphocytes in the immunotherapy era. Cell Mol Immunol. 2021;18(4):842-859.

2. Salgado R, et al. The evaluation of tumor-infil- trating lymphocytes (TILs) in breast cancer: recommendations by an International TILs Working Group 2014. Ann Oncol. 2015;26(2):259-271.

3. Buisseret L, et al. Tumor-infiltrating lymphocyte composition, organization and PD-1/ PD-L1 expression are linked in breast cancer. Oncoimmunology. 2017;6(1):e1257452.

4. Gu-Trantien C, et al. CD4+ follicular helper T cell 
infiltration predicts breast cancer survival. J Clin Invest. 2013;123(7):2873-2892.

5. Gago da Graca C, et al. Tertiary lymphoid structures: diversity in their development, composition, and role. JImmunol. 2021;206(2):273-281.

6. Sautes-Fridman C, et al. Tertiary lymphoid structures and B cells: Clinical impact and therapeutic modulation in cancer. Semin Immunol. 2020;48:101406.

7. Silina K, et al. Germinal centers determine the prognostic relevance of tertiary lymphoid structures and are impaired by corticosteroids in lung squamous cell carcinoma. Cancer Res. 2018;78(5):1308-1320.

8. Kroeger DR, et al. Tumor-infiltrating plasma cells are associated with tertiary lymphoid structures, cytolytic T-cell responses, and superior prognosis in ovarian cancer. Clin Cancer Res. 2016;22(12):3005-3015.

9. De Silva P, et al. Active and quiescent tertiary lymphoid structures, differentiated using FOXP1 expression, play a role in immunity to breast cancer. Paper presented at: AACR Special Conference on Tumor Immunology and Immunotherapy; November 17-20, 2019; Boston, Massachusetts, USA.

10. Cabrita R, et al. Tertiary lymphoid structures improve immunotherapy and survival in melanoma. Nature. 2020;577(7791):561-565.

11. Helmink BA, et al. B cells and tertiary lymphoid structures promote immunotherapy response. Nature. 2020;577(7791):549-555.

12. Petitprez F, et al. B cells are associated with survival and immunotherapy response in sarcoma. Nature. 2020;577(7791):556-560.

13. Posch F, et al. Maturation of tertiary lymphoid structures and recurrence of stage II and III colorectal cancer. Oncoimmunology. 2018;7(2):e1378844.

14. Gu-Trantien C, et al. CXCL13-producing TFH cells link immune suppression and adaptive memory in human breast cancer. JCI Insight . 2017;2(11):91487.

15. Crotty S. T follicular helper cell biology: a decade of discovery and diseases. Immunity. 2019;50(5):1132-1148.

16. Rao DA, et al. Pathologically expanded periphera T helper cell subset drives $B$ cells in rheumatoid arthritis. Nature. 2017;542(7639):110-114.

17. Gu-Trantien C, Willard-Gallo K. PD- ${ }^{\text {hi }}$ CXCR5 $\mathrm{CD} 4^{+} \mathrm{T}_{\mathrm{FH}}$ cells play defense in cancer and offense in arthritis. Trends Immunol. 2017;38(12):875-878.

18. Thommen DS, et al. A transcriptionally and functionally distinct $\mathrm{PD}-\mathrm{1}^{+} \mathrm{CD} 8^{+} \mathrm{T}$ cell pool with predictive potential in non-small-cell lung cancer treated with PD-1 blockade. Nat Med. 2018;24(7):994-1004.

19. Workel HH, et al. A transcriptionally distinct $\mathrm{CXCL}^{2} 3^{+} \mathrm{CD} 103^{+} \mathrm{CD} 8^{+} \mathrm{T}$-cell population is associated with B-cell recruitment and neoantigen load in human cancer. Cancer Immunol Res. 2019;7(5):784-796.

20. Havenar-Daughton C, et al. CXCL13 is a plasma biomarker of germinal center activity. Proc Natl Acad Sci U S A. 2016;113(10):2702-2707.

21. Schiffer L, et al. CXCL13 as a new biomarker of systemic lupus erythematosus and lupus nephritis - from bench to bedside? Clin Exp Immunol.
2015;179(1):85-89.

22. Bindea G, et al. Spatiotemporal dynamics of intratumoral immune cells reveal the immune landscape in human cancer. Immunity. 2013;39(4):782-795

23. Garaud S, et al. Tumor infiltrating B-cells signal functional humoral immune responses in breast cancer. JCI Insight. 2019;5:129641.

24. Loi S, et al. Tumor infiltrating lymphocytes are prognostic in triple negative breast cancer and predictive for trastuzumab benefit in early breast cancer: results from the FinHER trial. Ann Oncol. 2014;25(8):1544-1550.

25. Sage PT, Sharpe AH. The multifaceted functions of follicular regulatory T cells. Curr Opin Immunol. 2020;67:68-74.

26. Wang L, et al. Mbd2 promotes foxp3 demethylation and T-regulatory-cell function. Mol Cell Biol. 2013;33(20):4106-4115

27. Lienart $S$, et al. Structural basis of latent TGF- $\beta 1$ presentation and activation by GARP on human regulatory T cells. Science. 2018;362(6417):952-956.

28. Sayin I, et al. Spatial distribution and function of $\mathrm{T}$ follicular regulatory cells in human lymph nodes. J Exp Med. 2018;215(6):1531-1542.

29. Garaud S, Gu-Trantien C, et al. A simple and rapid protocol to non-enzymatically dissociate fresh human tissues for the analysis of infiltrating lymphocytes. JVis Exp. 2014;(94):52392.

30. Duhen T, et al. Co-expression of CD39 and CD103 identifies tumor-reactive CD8 T cells in human solid tumors. Nat Commun. 2018;9(1):2724.

31. Sade-Feldman M, et al. Defining T cell states associated with response to checkpoint immunotherapy in melanoma. Cell. 2018;175(4):998-1013.

32. Hendry S, et al. Assessing tumor-infiltrating lymphocytes in solid tumors: a practical review for pathologists and proposal for a standardized method from the international immuno-oncology biomarkers working group: part 2: TILs in melanoma, gastrointestinal tract carcinomas, nonsmall cell lung carcinoma and mesothelioma, endometrial and ovarian carcinomas, squamous cell carcinoma of the head and neck, genitourinary carcinomas, and primary brain tumors. $A d v$ Anat Pathol. 2017;24(6):311-335.

33. Hendry S, et al. Assessing tumor-infiltrating lymphocytes in solid tumors: a practical review for pathologists and proposal for a standardized method from the international immunooncology biomarkers working group: part 1: assessing the host immune response, TILs in invasive breast carcinoma and ductal carcinoma in situ, metastatic tumor deposits and areas for further research. Adv Anat Pathol. 2017;24(5):235-251.

34. Garaud S, et al. Antigen specificity and clinical significance of IgG and IgA autoantibodies produced in situ by tumor-infiltrating B cells in breast cancer. Front Immunol. 2018;9:2660.

35. Jacquelot $\mathrm{N}$, et al. Tertiary lymphoid structures and B lymphocytes in cancer prognosis and response to immunotherapies. Oncoimmunology. 2021;10(1):1900508.

36. Jia $\mathrm{Y}$, et al. Impaired function of $\mathrm{CD} 4+\mathrm{T}$ follicular helper (Tfh) cells associated with hepatocellular carcinoma progression. PLoS One. 2015;10(2):e0117458.
37. Ng KW, et al. Somatic mutation-associated $\mathrm{T}$ follicular helper cell elevation in lung adenocarcinoma. Oncoimmunology. 2018;7(12):e1504728.

38. Qian G, et al. Thyroid cancer metastasis is associated with an overabundance of defective follicular helper T cells. APMIS. 2020;128(8):487-496.

39. Solinas C, et al. Immune checkpoint molecules on tumor-infiltrating lymphocytes and their association with tertiary lymphoid structures in human breast cancer. Front Immunol. 2017;8:1412.

40. Solinas C, et al. BRCA gene mutations do not shape the extent and organization of tumor infiltrating lymphocytes in triple negative breast cancer. Cancer Lett. 2019;450:88-97.

41. Niessl J, et al. Persistent expansion and Th1-like skewing of HIV-specific circulating $\mathrm{T}$ follicular helper cells during antiretroviral therapy. EBioMedicine. 2020;54:102727.

42. Martin-Gayo E, et al. Immunological fingerprints of controllers developing neutralizing HIV-1 antibodies. Cell Rep. 2020;30(4):984-996.

43. Zhang J, et al. Spike-specific circulating T follicular helper cell and cross-neutralizing antibody responses in COVID-19-convalescent individuals. Nat Microbiol. 2021;6(1):51-58.

44. Kaneko N, et al. Loss of Bcl-6-expressing T follicular helper cells and germinal centers in COVID19. Cell. 2020;183(1):143-157.

45. Takeshita M, et al. Multi-dimensional analysis identified rheumatoid arthritis-driving pathway in human T cell. Ann Rheum Dis. 2019;78(10):1346-1356.

46. Yoshitomi H. CXCL13-producing PD- $1^{\text {hi }}$ CXCR5 helper $\mathrm{T}$ cells in chronic inflammation. Immunol Med. 2020;43(4):156-160.

47. Le KS, et al. CXCR5 and ICOS expression identifies a CD8 T-cell subset with $\mathrm{T}_{\mathrm{FH}}$ features in Hodgkin lymphomas. Blood Adv. 2018;2(15):1889-1900.

48. Wirsing AM, et al. Characterisation and prognostic value of tertiary lymphoid structures in oral squamous cell carcinoma. BMC Clin Pathol. 2014;14:38.

49. McGranahan N, et al. Clonal neoantigens elicit $\mathrm{T}$ cell immunoreactivity and sensitivity to immune checkpoint blockade. Science. 2016;351(6280):1463-1469.

50. Zhang Y, et al. The clinical impact of ICOS signal in colorectal cancer patients. Oncoimmunology. 2016;5(5):e1141857.

51. Velu V, et al. Thl 1 cells in germinal centers during chronic HIV/SIV infection. Front Immunol. 2018;9:1272.

52. Valentine KM, Hoyer KK. CXCR5+ CD8 T cells: protective or pathogenic? Front Immunol. 2019;10:1322.

53. Kalathil S, et al. Higher frequencies of GARP $(+)$ CTLA-4(+)Foxp3(+) T regulatory cells and myeloid-derived suppressor cells in hepatocellular carcinoma patients are associated with impaired T-cell functionality. Cancer Res. 2013;73(8):2435-2444.

54. Downs-Canner S, et al. Suppressive IL-17A ${ }^{+}$ Foxp $^{+}$and ex-Th17 IL-17A ${ }^{\text {neg }}$ Foxp $^{+} \mathrm{T}_{\text {reg }}$ cells are a source of tumour-associated $\mathrm{T}_{\text {reg }}$ cells. Nat Commun. 2017;8:14649.

55. Salem M, et al. GARP dampens cancer immunity by sustaining function and accumulation of regulatory $\mathrm{T}$ cells in the colon. Cancer Res. 
2019;79(6):1178-1190.

56. Coraglia A, et al. Common variable immunodeficiency and circulating TFH. J Immunol Res. 2016;2016:4951587.

57. Xing $\mathrm{M}$, et al. Induction of peripheral blood $\mathrm{T}$ follicular helper cells expressing ICOS correlates with antibody response to hepatitis B vaccination. J Med Virol. 2020;92(1):62-70.

58. Solinas $\mathrm{C}$, et al. The rationale behind targeting the ICOS-ICOS ligand costimulatory path- way in cancer immunotherapy. ESMO Open. 2020;5(1):e000544.

59. Chamoto K, et al. Role of PD-1 in immunity and diseases. Curr Top Microbiol Immunol. 2017;410:75-97.

60. Shi J, et al. PD-1 controls follicular T helper cell positioning and function. Immunity. 2018;49(2):264-274.

61. Hollern DP, et al. B cells and T follicular helper cells mediate response to checkpoint inhibitors in high mutation burden mouse models of breast cancer. Cell. 2019;179(5):1191-1206.

62. Zhang L, et al. Lineage tracking reveals dynamic relationships of $\mathrm{T}$ cells in colorectal cancer. Nature. 2018;564(7735):268-272.

63. Morita R, et al. Human blood CXCR5(+) $\mathrm{CD} 4(+) \mathrm{T}$ cells are counterparts of $\mathrm{T}$ follicular cells and contain specific subsets that differentially support antibody secretion. Immunity. 2011;34(1):108-121. 